\title{
Broadband Tunable Mid-infrared Plasmon Resonances in Cadmium Oxide Nanocrystals Induced by Size-dependent Nonstoichiometry
}

Zeke Liu, ${ }^{1,2, \perp}$ Yaxu Zhong, ${ }^{2, \perp}$ Ibrahim Shafei, ${ }^{2}$ Soojin Jeong, ${ }^{2}$ Liguang Wang, ${ }^{3}$ Hoai T. Nguyen, ${ }^{3}$ Cheng-Jun Sun, ${ }^{4}$ Tao Li, ${ }^{* 3,4}$ Jun Chen, ${ }^{2}$ Lei Chen, ${ }^{1,2}$ Yaroslav Losovyj, ${ }^{2}$ Xinfeng Gao, ${ }^{2}$ Wanli $\mathrm{Ma},{ }^{* 1}$ Xingchen $\mathrm{Ye}^{* 2}$

${ }^{1}$ Jiangsu Key Laboratory for Carbon-Based Functional Materials \& Devices, Institute of Functional Nano \& Soft Materials (FUNSOM), Joint International Research Laboratory of CarbonBased Functional Materials and Devices, Soochow University, 199 Ren'ai Road, Suzhou, 215123, Jiangsu, China.

${ }^{2}$ Department of Chemistry, Indiana University, 800 East Kirkwood Avenue, Bloomington, Indiana 47405, USA.

${ }^{3}$ Department of Chemistry and Biochemistry, Northern Illinois University, 1425 W. Lincoln Hwy., DeKalb, IL, 60115, USA.

${ }^{4}$ X-ray Science Division, Argonne National Laboratory, 9700 South Cass Avenue, Lemont, Illinois 60439, USA.

Correspondence should be addressed to T.L. (email: taoli@anl.gov), W.M. (email: wlma@suda.edu.cn) or to X.Y. (email: xingye@indiana.edu).

${ }^{\perp}$ These authors contributed equally to this work. 


\section{Contents}

Experimental details ....................................................................................................................... S3

Text

Drude fitting of LSPR extinction spectra ................................................................................ S4

Text, Figure 59

Size-dependent nonstoichiometry model of CdO NCs. ..................................................................S5

Text, Figure S1,S2 Table S1

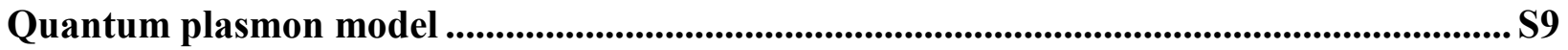

Text, Figure 520

Hybrid size-dependent nonstoichiometry and quantum plasmon model............................... S9

Text, Figure S21

CdO NCs synthesized using different additives and reaction parameters ........................... S10

Figure S10-S15, Table S2,S4

Size-controlled synthesis of $\mathrm{CdO}$ NCs by varying the HMDS/Cd ratio S11

Figure S3-S9, Table S3

X-ray absorption spectroscopy of CdO NCs and fitting results.............................................S21

Figure S17, Table S5

XPS analysis of CdO NCs....................................................................................................... S24

Figure S18-S19, Table S6

EPR spectra and thermogravimetric analysis of CdO NCs.....................................................S27

Figure S22-S24 


\section{Experimental details and data analysis}

Chemicals: Cadmium(II) acetylacetonate $\left(\mathrm{Cd}(\mathrm{acac})_{2}, \geq 99.9 \%\right)$, oleic acid (OLAC, 90\%), 1-octadecene (ODE, 90\%), lithium bis(trimethylsilyl)amide (LiHMDS, 97\%), tris(trimethylsilyl)silane (TTMSS, 97\%), lithium triethylborohydride (superhydride, $1.0 \mathrm{M}$ solution in tetrahydrofuran), $\mathrm{n}$ butyllithium (BuLi, 1.6 M in hexanes), borane tert-butylamine complex (TBAB, 97\%), tetrachloroethylene (TCE, 99\%), carbon tetrachloride $\left(\mathrm{CCl}_{4}, 99.9 \%\right)$ and carbon disulfide $\left(\mathrm{CS}_{2}, 99 \%\right)$ were purchased from Sigma-Aldrich. Bis(trimethylsilyl)amine (HMDS, 98\%) and trioctylphosphine oxide (TOPO, 99\%) were purchased from Acros Organics. Indium acetate $\left(\operatorname{In}(\mathrm{ac})_{3}, 99.99 \%\right)$ was purchased from Alfa Aesar. Anhydrous solvents were obtained from a custom-built solvent purification system. Unless otherwise specified, reagents were used as received without further purification.

Synthesis of CdO nanocrystals. All NC syntheses were carried out under nitrogen atmosphere using standard Schlenk line techniques. In a typical reaction, $1.5 \mathrm{mmol}$ of $\mathrm{Cd}(\mathrm{acac})_{2}, 7.5 \mathrm{mmol}$ of OLAC and $50 \mathrm{~mL}$ of ODE were loaded into a $100 \mathrm{~mL}$ three-neck flask. After degassing under vacuum for one hour at $100{ }^{\circ} \mathrm{C}$, a certain amount of additive diluted with vacuum-dried ODE was injected. The reaction mixture was then heated rapidly to its reflux temperature $\left(\sim 316^{\circ} \mathrm{C}\right)$. The color of the solution turned dark brown following 5-30 min of refluxing, indicating the formation of $\mathrm{CdO}$ NCs. The reaction flask was air-cooled to room temperature $30 \mathrm{~min}$ after the initial color change, and NCs were isolated by precipitation with isopropanol and centrifugation at $6000 \mathrm{rpm}$ for $5 \mathrm{~min}$. The NCs were re-dispersed in $20 \mathrm{~mL}$ of hexane and centrifuged at $3000 \mathrm{rpm}$ for $3 \mathrm{~min}$ to remove insoluble aggregates and metallic $\mathrm{Cd} \mathrm{NC}$ byproducts. For large $\mathrm{CdO} \mathrm{NCs}$ (average diameter $>30 \mathrm{~nm}$ ), removal of Cd NCs were done by re-dispersion in $20 \mathrm{~mL}$ of chloroform followed by centrifugation at $1000 \mathrm{rpm}$ for $3 \mathrm{~min}$. After the second round of isopropanol precipitation and centrifugation, $\mathrm{CdO} \mathrm{NCs}$ were stored as solids in a nitrogen-filled glovebox. The size-selective precipitation process described in Figure 3 was carried out in a nitrogen-filled glovebox to minimize possible LSPR peak shifts due to surface oxidation and/or moisture-material interactions.

Structural and optical characterization. Low-magnification TEM images were acquired on a JEOL JEM 1400 plus microscope with a $\mathrm{LaB}_{6}$ filament operating at $120 \mathrm{kV}$. TEM samples were prepared by drop-casting $\sim 10 \mu \mathrm{L}$ of NC solution onto a 300-mesh carbon-coated copper grid (Ted Pella). High-resolution TEM imaging was carried out on a $300 \mathrm{kV}$ JEOL JEM 3200FS TEM equipped with a Gatan 4k $\times$ 4k Ultrascan 4000 camera. Sample was prepared using holey 400mesh $\mathrm{Cu}$ grids coated with ultrathin carbon films (Ted Pella). EDX analysis was conducted with an X-Max $50 \mathrm{~mm}^{2}$ silicon drift detector (Oxford Instruments) interfaced to a Carl Zeiss Auriga SEM via the AZtec software package. For SEM-EDX analysis, NCs were thoroughly cleaned with the precipitation-redispersion process for 4-6 times before being deposited onto a silicon substrate. Solution-phase extinction spectra were recorded either on a Varian Cary 5000 spectrometer or with a Bruker Vertex 70 Fourier-transform infrared spectroscopy (FTIR) spectrometer in transmission mode using a Harrick demountable liquid cell.

X-ray diffraction. Powder XRD patterns were collected on a PANalytical Empyrean diffractometer with a copper target. The size of coherently scattering regions, or the so-called coherence length $L$, can be estimated by using the Debye-Scherrer equation: $L=\frac{K \lambda}{\beta \cos (\theta)} . K$ is a dimensionless shape factor that equals to 0.9 for spherical NCs. ${ }^{1,2} \lambda$ is incident $\mathrm{x}$-ray wavelength $(0.154 \mathrm{~nm})$. 
$\theta$ is the Bragg angle and $\beta$ is the full-width at half-maximum (fwhm) of the corresponding XRD peak. The fwhm values were corrected for instrumental broadening by, $\beta=\left(\beta_{\text {exp }}^{2}-\beta_{\text {ins }}^{2}\right)^{1 / 2}$, where $\beta_{\text {exp }}$ is the experimental fwhm of diffraction peak and $\beta_{\text {ins }}$ is the instrumental broadening (0.02 degree). According to previous rigorous treatment taking into account the distribution of coherent length for a spherical NC, the average NC diameter $D$ can be expressed in terms of $L$ as: $D=\frac{4}{3} L{ }^{2-4}$

X-ray photoelectron spectroscopy. XPS analysis was performed on a PHI VersaProbe II instrument equipped with a focused monochromatic Al K(alpha) source. The instrument base pressure was about $8 \times 10^{-10}$ Torr. A beam size of about 200 micrometer and an X-ray power of $50 \mathrm{~W}$ at 15 $\mathrm{keV}$ was used for all experiments. The $\mathrm{C} 1 \mathrm{~s}$ peak calibrated at $284.4 \mathrm{eV}$ was used as an internal standard for the binding energy scale. NCs were thoroughly cleaned with the precipitation-redispersion process for 4-6 times before being deposited onto an aluminum foil substrate.

X-ray absorption spectroscopy (X-ray absorption near-edge structure (XANES) and Extended X-ray absorption fine structure (EXAFS)). X-ray absorption spectroscopy experiments were performed on Cd-K edge at beamline 20-BM-B of Advanced Photon Source (APS) at Argonne National Laboratory (ANL). CdO NCs were pelleted together with boron nitride to obtain the optimal sample thickness for transmission mode experiments. A cadmium foil spectrum was acquired simultaneously during each measurement for energy calibration. The X-ray absorption data were processed using established methods available with the Athena software. The normalized EXAFS were Fourier transformed from energy related k-space to real space and these spectra were weighted by $\mathrm{k}^{2}$ to compensate for damping of oscillation at high $\mathrm{k}$. The EXAFS data were fitted with the Artemis software to gain information on the local environment of cadmium sites. First, the amplitude reduction factor $\left(\mathrm{S}_{0}^{2}\right)$ was obtained as 0.98 by fitting EXAFS data of the cadmium foil, and this parameter was kept fixed during spectral fitting of different NC samples. Our EXAFS fittings took into account contributions from the first coordination shell of the neighboring oxygen atoms (i.e., $\mathrm{Cd}-\mathrm{O}$ ) and the second coordination shell of the cation sites (i.e., $\mathrm{Cd}-\mathrm{Cd}$ ).

Electron paramagnetic resonance spectroscopy. EPR spectra were recorded on a Bruker EMX X-band EPR spectrometer with an ER 4119 HS cavity using $100 \mathrm{kHz}$ field modulation (modulation amplitude: $6 \mathrm{G}$ ). Individual EPR tubes were filled with $0.5 \mathrm{~mL}$ of solution inside a glovebox and were placed at the same position inside the resonant cavity during EPR spectral acquisition. The magnetic field $\left(B_{0}\right)$ can be converted to $g$ value according to $g=h v / \beta B_{0}$. where $h$ is the Planck's constant $\left(6.626 \times 10^{-34} \mathrm{~J} \cdot \mathrm{s}\right), v$ is the frequency of the radiation $\left(9.800475 \times 10^{9} \mathrm{~Hz}\right)$ and $\beta$ is the Bohr magneton $\left(9.274 \times 10^{-28} \mathrm{~J} \cdot \mathrm{G}^{-1}\right)$.

Nuclear magnetic resonance (NMR). ${ }^{1} \mathrm{H}-\mathrm{NMR}$ measurements were performed on a Varian Inova $600 \mathrm{MHz}$ spectrometer. Samples were dissolved in deuterated chloroform and chemical shifts were referenced to the residual chloroform signal at $7.26 \mathrm{ppm}$.

Drude fitting of LSPR extinction spectra. Drude fitting of LSPR peaks was performed by using a custom-written MATLAB script. ${ }^{5}$ The absorbance $(A)$ of NC solutions is determined by the Beer-Lambert law: 


$$
A=\frac{N \sigma_{\mathrm{A}} l}{\ln (10)}
$$

where $N$ is the NC concentration in solution, and $l$ is the path length of the cuvette. According to the Mie theory, ${ }^{6}$ the absorption cross section $\left(\sigma_{\mathrm{A}}\right)$ of spherical NCs is calculated as:

$$
\sigma_{\mathrm{A}}=4 \pi \mathbf{k} r^{3} \operatorname{Imag}\left(\frac{\varepsilon(\omega)-\varepsilon_{\mathrm{m}}}{\varepsilon(\omega)+2 \varepsilon_{\mathrm{m}}}\right)
$$

where $\mathbf{k}$ is the wave vector of the incident light and $\mathbf{k}=\sqrt{\varepsilon_{\mathrm{m}}} \omega / c, c$ is the speed of light, $r$ is the NC radius, $\varepsilon(\omega)$ is the dielectric function of NCs and $\varepsilon_{\mathrm{m}}$ is the dielectric constant of the medium (for TCE, $\varepsilon_{\mathrm{m}}=2.268$ ). The frequency-dependent dielectric function is given by

$$
\varepsilon_{(\omega)}=\varepsilon_{\infty}-\frac{\omega_{\mathrm{p}}{ }^{2}}{\left(\omega^{2}+\mathrm{i} \omega \Gamma\right)}
$$

where $\varepsilon_{\infty}$ is the high-frequency dielectric constant (for $\mathrm{CdO}, \varepsilon_{\infty}=5.5$ ), ${ }^{7} \omega_{\mathrm{p}}$ is the bulk plasma frequency, and $\Gamma$ is the free carrier damping constant. By fitting the LSPR absorption spectrum with the Drude model, $\omega_{\mathrm{p}}$ and $\Gamma$ can be retrieved. The free carrier concentration $N_{\mathrm{e}}$ is calculated as: ${ }^{8,9}$

$$
\omega_{\mathrm{p}}=\sqrt{\frac{N_{\mathrm{e}} e^{2}}{m^{*} \varepsilon_{0}}}, \quad N_{\mathrm{e}}=\frac{\omega_{\mathrm{p}}{ }^{2} m^{*} \varepsilon_{0}}{e^{2}}
$$

where $e$ is the elementary charge, $m^{*}$ is the effective mass of electrons (for $\mathrm{CdO}, m^{*}=0.27 m_{\mathrm{e}}$, where $m_{\mathrm{e}}$ is the electron rest mass), ${ }^{10}$ and $\varepsilon_{0}$ is the vacuum permittivity.

Size-dependent nonstoichiometry model of CdO NCs. The size-dependent nonstoichiometry model for $\mathrm{CdO} \mathrm{NCs}$ we propose here is similar to previous examples on rocksalt-type $\mathrm{PbS}$ and $\mathrm{PbSe}$ NCs. ${ }^{11,12}$ Individual CdO NCs are modelled as cuboctahedra expressing $\{100\}$ and $\{111\}$ facets on the surface (Figure S1). 

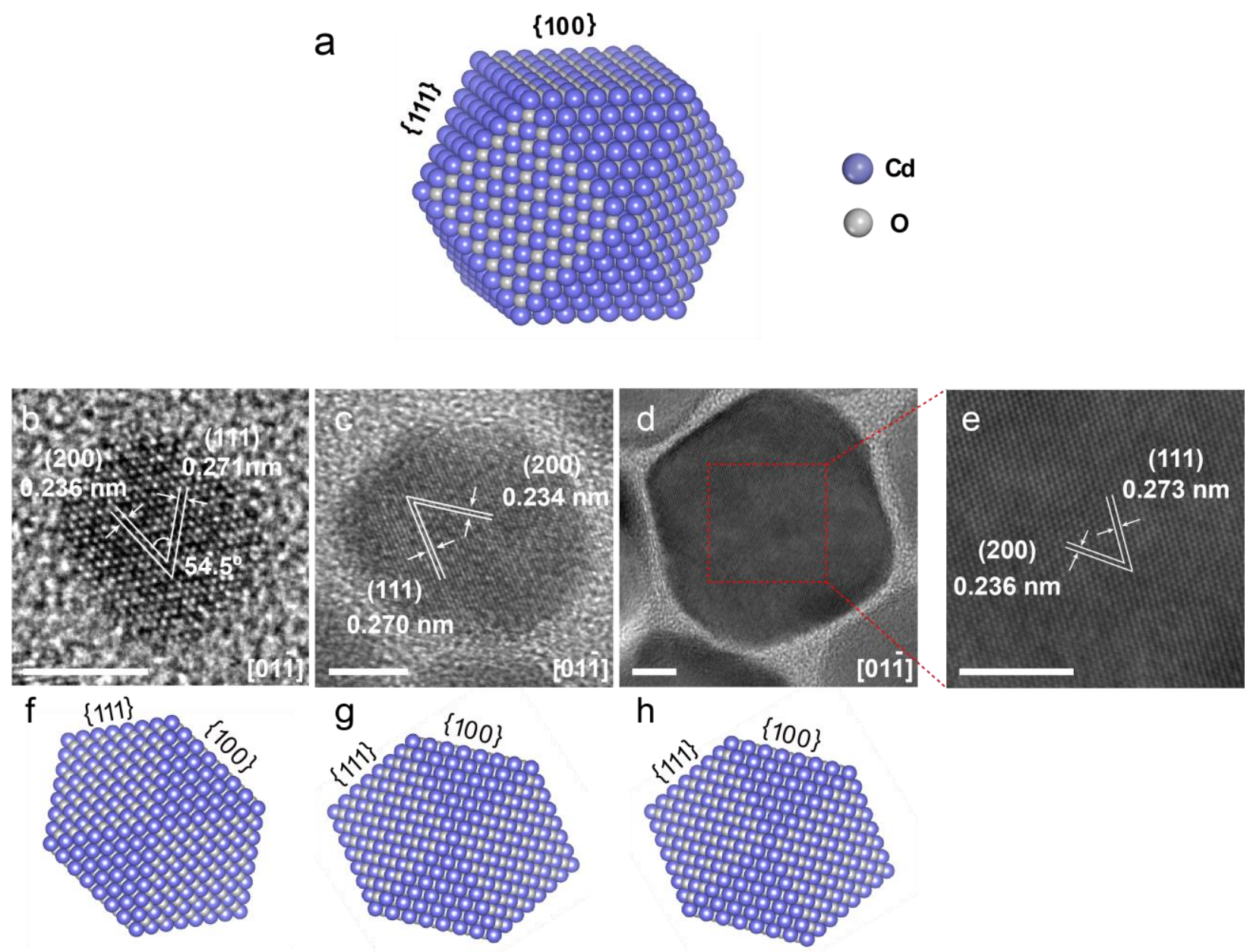

Figure S1. (a) Atomistic model of cuboctahedral CdO NCs. (b-e) HRTEM images of CdO NCs with an average diameter of $7.9 \mathrm{~nm}$ (b) $14.2 \mathrm{~nm}$ (c) and $28.9 \mathrm{~nm}$, respectively, and (f-h) corresponding atomistic models. Scale bars: $5 \mathrm{~nm}$.

The $\{100\}$ surfaces have a 1:1 molar ratio between $\mathrm{Cd}$ and $\mathrm{O}$ atoms, whereas the $\{111\}$ surfaces are assumed to be terminated by $\mathrm{Cd}^{2+}$ ions that are passivated by the oleate ligand. This structural model suggests a cadmium-rich stoichiometry that depends on the size of CdO NCs. The number of excess $\mathrm{Cd}^{2+}(N)$ is calculated as follows:

$$
N=\frac{\sqrt{3}}{4} \times\left(\frac{d}{2}\right)^{2} \times 8 \times \frac{1}{2} \times A=\frac{\sqrt{3}}{4} d^{2} A
$$

Here $d$ is the diameter of cuboctahedral NCs, which is equal to the distance between two farthest vertices. The factor 8 in the above formula is due to the fact that there are $8\{111\}$ facets for a cuboctahedron. As illustrated in Figure S2, the factor $1 / 2$ derives from the fact that only $50 \%$ of $\{111\}$ surface $\mathrm{Cd}^{2+}$ are excessive ions leading to non-stoichiometry. 


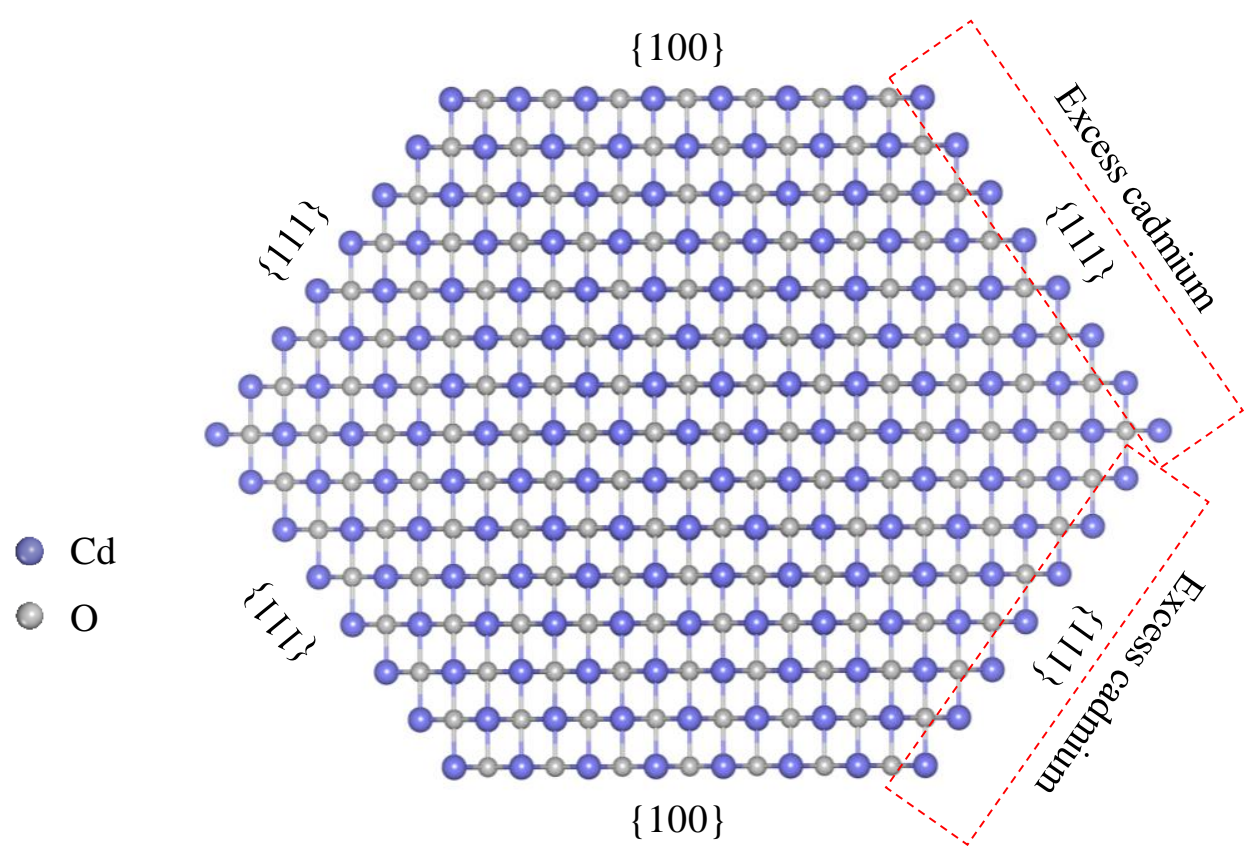

Figure S2. Two-dimensional schematic illustration of non-stoichiometric cuboctahedral CdO NCs.

The lattice constant of $\mathrm{CdO}$ is $a=4.6953 \AA$. $A$ is the density of $\mathrm{Cd}^{2+}$ ions on the $\{111\}$ facets:

$$
A=\frac{4 \sqrt{3}}{3 a^{2}}=10.4755 \mathrm{~nm}^{-2}
$$

The concentration $(D)$ of excess $\mathrm{Cd}^{2+}$ can be calculated by dividing the number of extra $\mathrm{Cd}^{2+}$ over NC volume,

$$
D=\frac{\frac{\sqrt{3}}{4} d^{2} A}{\frac{5 \sqrt{2}}{24} d^{3}}=\frac{3 \sqrt{6} A}{5 d}
$$

To validate the accuracy of $\mathrm{NC}$-size-dependent excess $\mathrm{Cd}^{2+}$ concentrations established with equations (5)-(6), we further evaluated the $\mathrm{Cd} / \mathrm{O}$ ratio based on atomistic models built with the software package VESTA. ${ }^{13}$ As detailed in Table $\mathrm{S} 1$, the difference in $\mathrm{Cd} / \mathrm{O}$ ratio derived from geometric (equations (5)-(6)) and atomistic (VESTA software) models is less than $1.5 \%$. 
Table S1. Comparison of Cd/O ratio calculated based on the geometric model (equations (5)-(6)) and the atomistic model (built with VESTA) of cuboctahedral CdO NCs.

\begin{tabular}{ccccc}
\hline \multirow{2}{*}{ NC diameter } & Model & $\begin{array}{c}\text { Total number of } \\
\text { Cd atoms }\end{array}$ & $\begin{array}{c}\text { Total number of O } \\
\text { atoms }\end{array}$ & Cd/O ratio \\
\hline \multirow{2}{*}{$5 \mathrm{~nm}$} & Atomistic & 1413 & 1290 & 1.09535 \\
& Geometric & 1537 & 1423 & 1.08011 \\
\multirow{2}{*}{$10 \mathrm{~nm}$} & Atomistic & 12431 & 11920 & 1.04287 \\
& Geometric & 11839 & 11386 & 1.03979 \\
\multirow{2}{*}{$15 \mathrm{~nm}$} & Atomistic & 43287 & 42136 & 1.02732 \\
& Geometric & 39446 & 38426 & 1.02654 \\
& Atomistic & 94611 & 92690 & 1.02072 \\
& Geometric & 92897 & 91082 & 1.01993 \\
\hline
\end{tabular}

Free carriers of $\mathrm{CdO} \mathrm{NCs}$ include contributions from excess surface $\mathrm{Cd}^{2+}$ and oxygen vacancies within the interior of NCs. The total carrier concentration $\left(N_{\mathrm{e}}\right)$ of $\mathrm{CdO} \mathrm{NCs}$ can be retrieved via Drude fitting of experimental extinction spectra and results were summarized in Table S4. By performing a linear fit to the plot of carrier concentration $\left(N_{\mathrm{e}}\right)$ against excess $\mathrm{Cd}^{2+}$ concentration (D) calculated based on the size-dependent nonstoichiometry model (Figure 4b), the apparent "doping efficiency" due to $\mathrm{Cd}^{2+}$ is obtained from the best-fit slope. The "background" carrier density due to lattice oxygen vacancies is available from the y-intercept. The retrieved "doping efficiency" is $7.3 \%$ for these self-doped $\mathrm{CdO} \mathrm{NCs}$, which is slightly lower than aliovalently doped plasmonic metal oxide NCs (Table S7). We note that the $7.3 \%$ doping efficiency is an estimated average that is subject to influences by several factors. Firstly, deviations from the perfect cuboctahedral shape for as-synthesized $\mathrm{CdO}$ NCs can lead to variations in the relative proportions of $\{100\}$ and $\{111\}$ facets on NC surface, which may alter the effective doping efficiency. Secondly, the almost inevitable size polydispersity of NCs may broaden the distribution of doping efficiency values within an ensemble of NCs.

The carrier concentration within NC interior $\left(N_{0}\right)$ is determined to be $8.86 \times 10^{19} \mathrm{~cm}^{-3}$ (Figure $4 \mathrm{~b}$ ), which is in excellent agreement with previous studies on bulk $\mathrm{CdO}$ film $\left(6.6 \times 10^{19} \mathrm{~cm}^{-3}\right) .{ }^{14}$ This result provides further support for the effectiveness of our size-dependent nonstoichiometry model in capturing the essential physics of LSPR in CdO NCs. The extracted doping efficiency $(x)$ from excess surface $\mathrm{Cd}^{2+}$ and $N_{0}$ are used to calculate the total carrier concentration $\left(N_{\mathrm{e}}\right)$ of CdO NCs:

$$
N_{e}=2 x D+N_{0}=\frac{6 \sqrt{6} A}{5 d} x+N_{0}
$$

The results are plotted in Figure 4a, which shows good agreement with a large set of experimental data points from $\mathrm{CdO}$ NCs synthesized with different additives. 
Lastly, the charge state of some oxygen vacancy sites likely changes from $V_{\mathrm{O}}{ }^{*}$ to $V_{\mathrm{O}}{ }^{\circ}$ as $\mathrm{NC}$ size decreases, as indicated by the EPR measurement results. This possibility is not captured by our size-dependent nonstoichiometry model because we postulate that $V_{\mathrm{O}}{ }^{*}$ remains the dominant form of oxygen vacancy sites given its thermodynamically favorable formation energy in $\mathrm{CdO} .{ }^{15}$

Quantum plasmon model. To assess the potential contribution of quantum effects to the LSPR property in our CdO NCs, we used quantum plasmon model proposed by Zhang et al.: ${ }^{16-18}$

$$
\omega_{\text {sp }}^{2}=\omega_{\text {classical }}^{2}+\left(\frac{4 y \hbar^{2}}{m^{*}}\right)^{2} \times\left(\frac{1}{d}\right)^{4}
$$

where $\omega_{\text {sp }}$ is the measured LSPR energy, $\omega_{\text {classical }}$ is the corresponding material-specific classical LSPR energy (observed for $r \rightarrow \infty$ ), $m^{*}$ is the effective mass of conduction electrons, $y$ is a proportionality constant and $d$ is the diameter of CdO NCs. $\hbar$ is the reduced Plank constant. A linear regression was performed on the plot of $\omega_{\mathrm{sp}}{ }^{2}$ versus $\frac{1}{d^{4}}$. The poor fitting shown in Figure S20 indicates that the size-dependent LSPR feature in CdO NCs cannot be accounted for by quantum plasmon effects alone.

Hybrid size-dependent nonstoichiometry and quantum plasmon model. We also evaluated the effectiveness of a hybrid size-dependent nonstoichiometry and quantum plasmon model. The LSPR energy $\left(\omega_{\mathrm{sp}}\right)$ is related to the bulk plasmon frequency $\left(\omega_{\mathrm{p}}\right) \mathrm{as}^{8,19}$

$$
\omega_{\mathrm{sp}}=\sqrt{\frac{\omega_{\mathrm{p}}^{2}}{\varepsilon_{\infty}+2 \varepsilon_{\mathrm{m}}}-\Gamma^{2}}
$$

For $\mathrm{CdO}, \Gamma$ has been shown to be relatively insignificant compared with the first term in the radicand, ${ }^{20,21}$ and therefore the equation can be simplified as

$$
\omega_{\mathrm{sp}}=\frac{\omega_{\mathrm{p}}}{\sqrt{\varepsilon_{\infty}+2 \varepsilon_{\mathrm{m}}}}
$$

The LSPR energy based on the size-dependent nonstoichiometry model is calculated as

$$
\omega_{\mathrm{sp}}=\sqrt{\frac{\left(x D+N_{0}\right) e^{2}}{\left(\varepsilon_{\infty}+2 \varepsilon_{\mathrm{m}}\right) m^{*} \varepsilon_{0}}}=\sqrt{\frac{\left(x \frac{6 \sqrt{6} A}{5 d}+N_{0}\right) e^{2}}{\left(\varepsilon_{\infty}+2 \varepsilon_{\mathrm{m}}\right) m^{*} \varepsilon_{0}}}
$$

The overall equation combining size-dependent nonstoichiometry and quantum plasmon effects can be written as follows:

$$
\omega_{\mathrm{sp}}^{2}=\frac{6 \sqrt{6} x A e^{2}}{5\left(\varepsilon_{\infty}+2 \varepsilon_{\mathrm{m}}\right) m^{*} \varepsilon_{0}} \times \frac{1}{d}+\frac{N_{0} e^{2}}{\left(\varepsilon_{\infty}+2 \varepsilon_{\mathrm{m}}\right) m^{*} \varepsilon_{0}}+\left(\frac{4 y \hbar^{2}}{m^{*}}\right)^{2} \times\left(\frac{1}{d}\right)^{4}
$$

Setting $\mathrm{c}=\frac{6 \sqrt{6} x A e^{2}}{5\left(\varepsilon_{\infty}+2 \varepsilon_{\mathrm{m}}\right) m^{*} \varepsilon_{0}}, \mathrm{~b}=\frac{N_{0} e^{2}}{\left(\varepsilon_{\infty}+2 \varepsilon_{\mathrm{m}}\right) m^{*} \varepsilon_{0}}, a=\left(\frac{4 y \hbar^{2}}{m^{*}}\right)^{2}$, equation (12) can be simplified as 


$$
\omega_{\mathrm{sp}}^{2}=\mathrm{c}\left(\frac{1}{d}\right)+\mathrm{b}+\mathrm{a}\left(\frac{1}{d}\right)^{4}
$$

Least-squares polynomial fit to the $\omega_{\mathrm{sp}}{ }^{2}$ versus $\frac{1}{d}$ plot returns the coefficient $a$ to be essentially zero (Figure S21). This result suggests that the quantum plasmon effect is unimportant, and the LSPR feature in these self-doped CdO NCs is well described by the size-dependent nonstoichiometry model.

From the extracted coefficient $\mathrm{c}=\frac{6 \sqrt{6} x \mathrm{Ae}^{2}}{5\left(\varepsilon_{\infty}+2 \varepsilon_{\mathrm{m}}\right) \mathrm{m}^{*} \varepsilon_{0}}=1.16016 \mathrm{eV}^{2} \mathrm{~nm}$, the doping efficiency is calculated to be $x=7.97 \%$. From the extracted value of coefficient $b=\frac{N_{0} e^{2}}{\left(\varepsilon_{\infty}+2 \varepsilon_{\mathrm{m}}\right) m^{*} \varepsilon_{0}}=0.04385 \mathrm{eV}^{2}$, the bulk carrier concentration $\left(N_{0}\right)$ is calculated to be $8.65 \times 10^{19} \mathrm{~cm}^{-3}$. Both values are very close to those retrieved from the size-dependent nonstoichiometry model.

Table S2. List of additives used in this work and their chemical structures.

\begin{tabular}{ll}
\hline Additive & Chemical structure \\
\hline HMDS & \\
TTMSS &
\end{tabular}



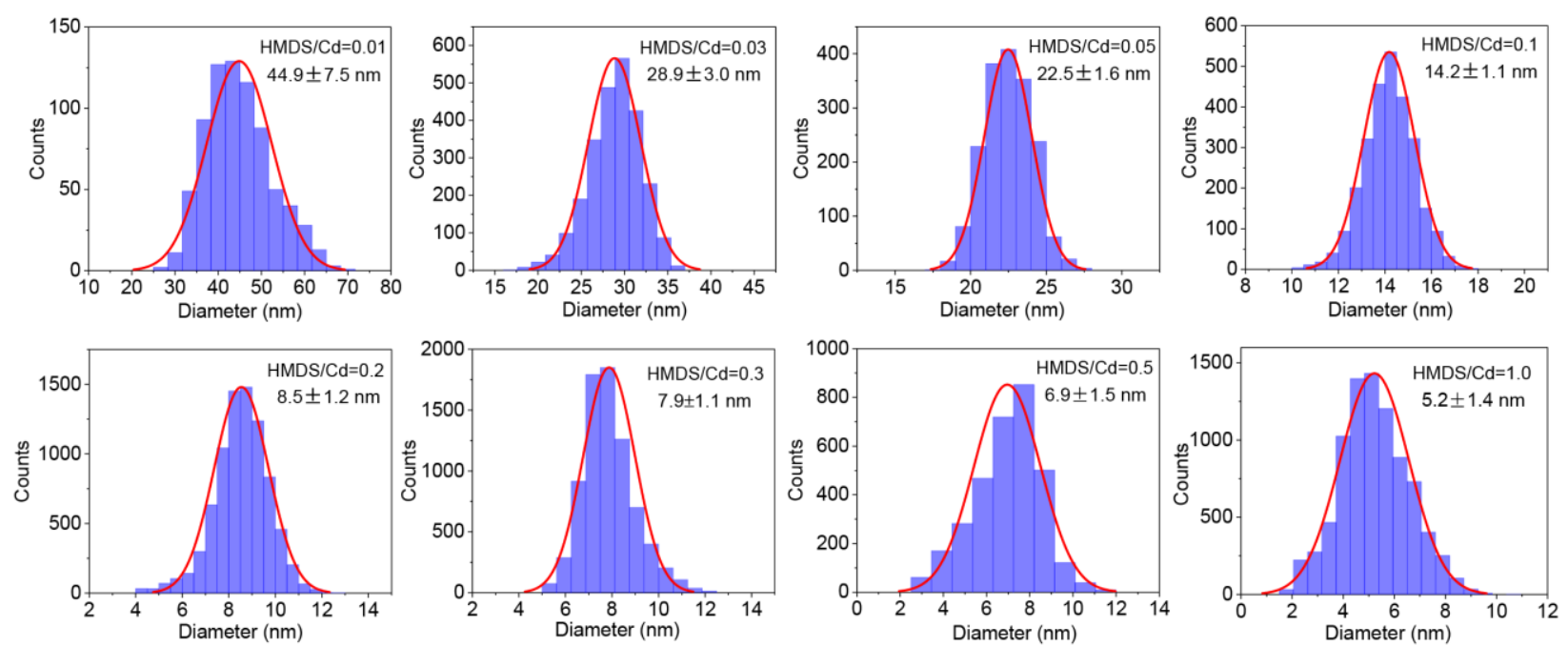

Figure S3. NC size distribution histograms determined from TEM images of CdO NCs synthesized using different HMDS/Cd molar ratios. The superimposed red curve on each histogram represents Gaussian fit to the distribution.

Table S3. Summary of average NC size determined from statistical analysis of TEM images and from Scherrer analysis of XRD patterns for CdO NCs synthesized using different HMDS/Cd molar ratios.

HMDS/Cd

(mole/mole)
Average diameter (TEM)

(nm)

44.5

28.9

22.5

14.2

8.5

6.9

5.2
Diameter (XRD)

(nm)

29.2

23.9

15.5

8.9

0.5

1

6.6

5.4 


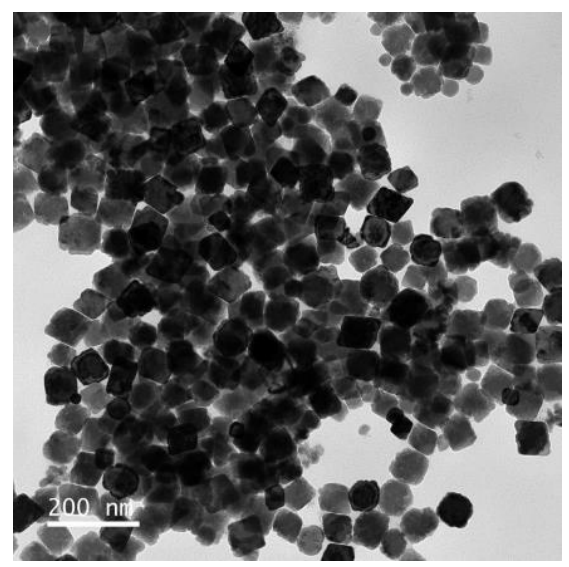

Figure S4. TEM image of CdO NCs synthesized in the absence of any additives.

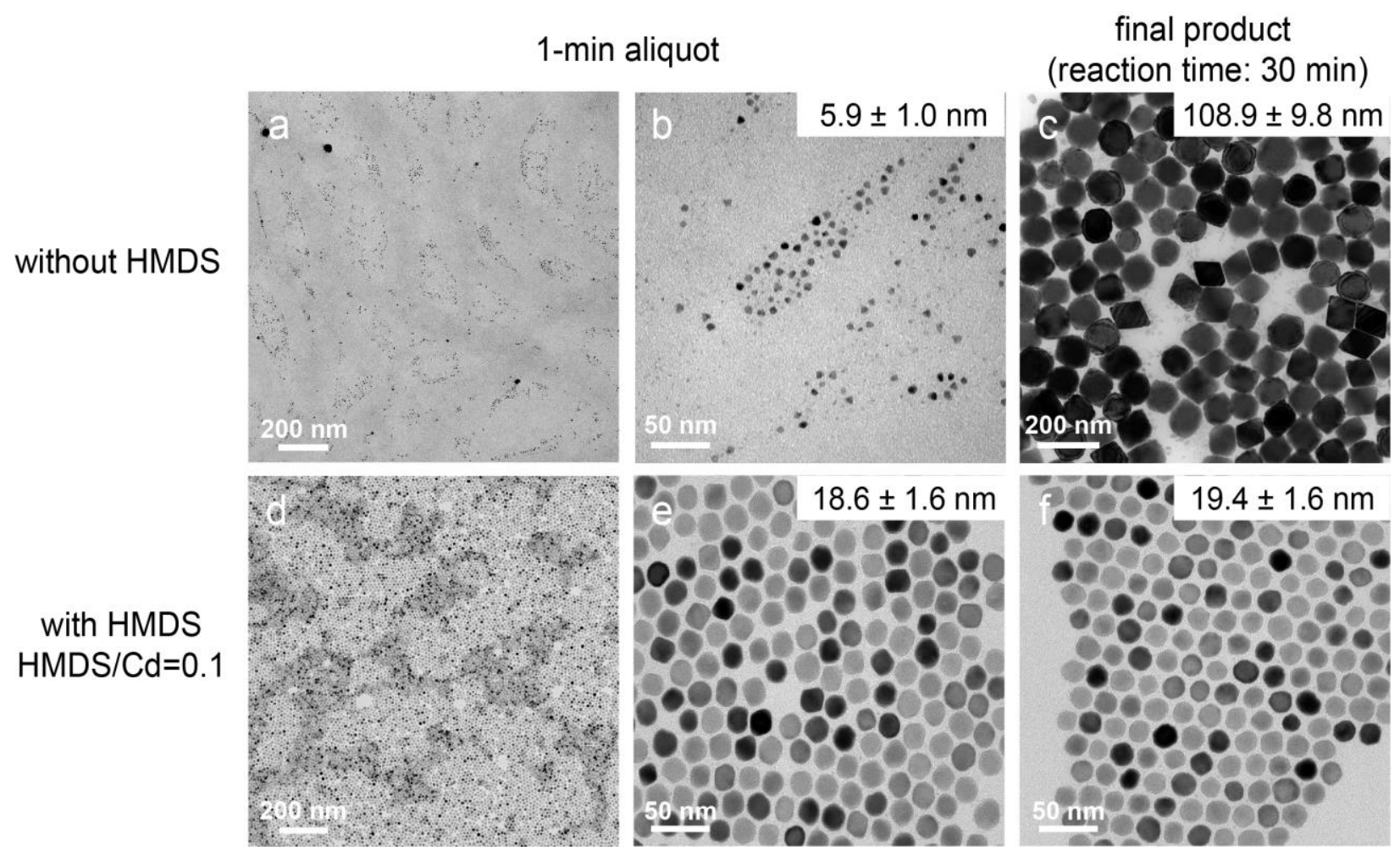

Figure S5. TEM images of CdO NCs isolated at different times from the reaction mixture (a,b,c) without and (d,e,f) with HMDS (HMDS/Cd=0.1) as the additive. Sample (a) and (d) were prepared by drop-casting NCs from solutions with the same concentration as the reaction mixture. The greater NC coverage shown in (d) indicates that nucleation was accelerated in the presence of HMDS. In the absence of HMDS, CdO NCs were ca. $5.9 \mathrm{~nm} 1$ minute after the initial color change of reaction solution and continued to grow, reaching nearly $110 \mathrm{~nm}$ after 30 minutes of reaction (Figure S5b, c). In contrast, CdO NCs synthesized with HMDS reached an average size of 18.6 $\mathrm{nm}$ within 1 minute following initial color change and further growth was found to be essentially negligible (Figure S5e, f). Based on these experimental data, we speculate that the addition of 
HMDS caused a rapid nucleation of NCs, depleting the vast majority of cadmium precursor within a short period of time after the reaction commences.

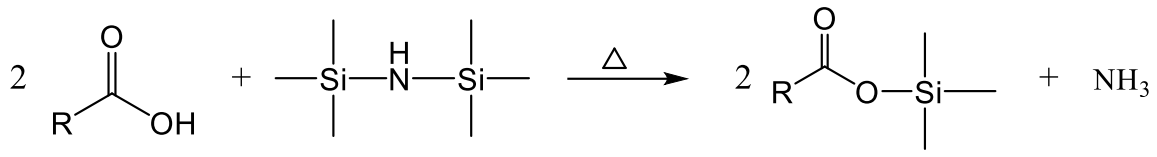

Scheme S1. Proposed reaction scheme between HMDS and OLAC. It has been shown that HMDS can react with acid to produce trimethylsilyl esters and gaseous ammonia in the presence of a catalyst or at elevated temperatures. ${ }^{22,23}$

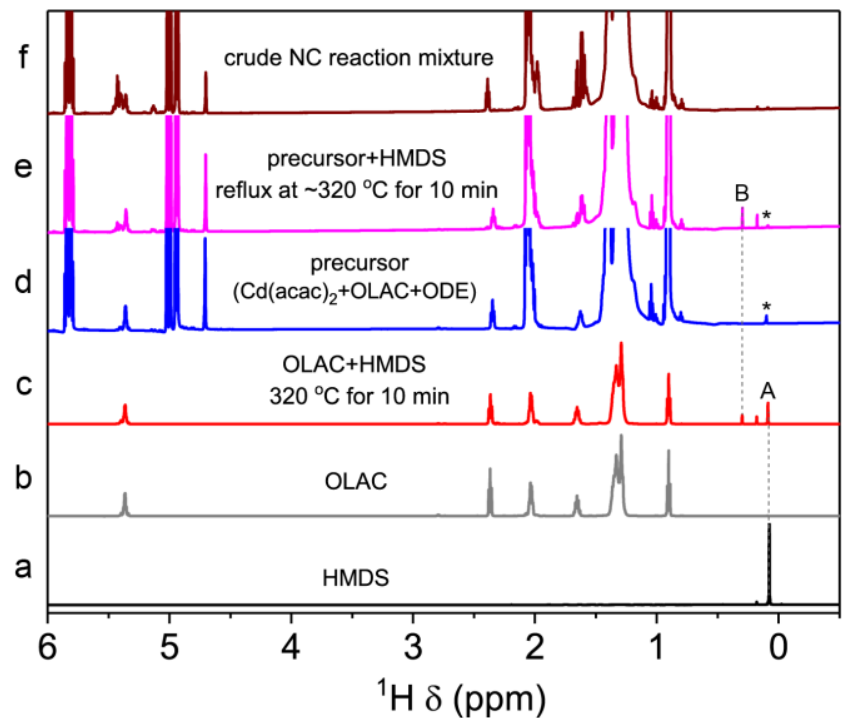

Peak A at $0.08 \mathrm{ppm}$

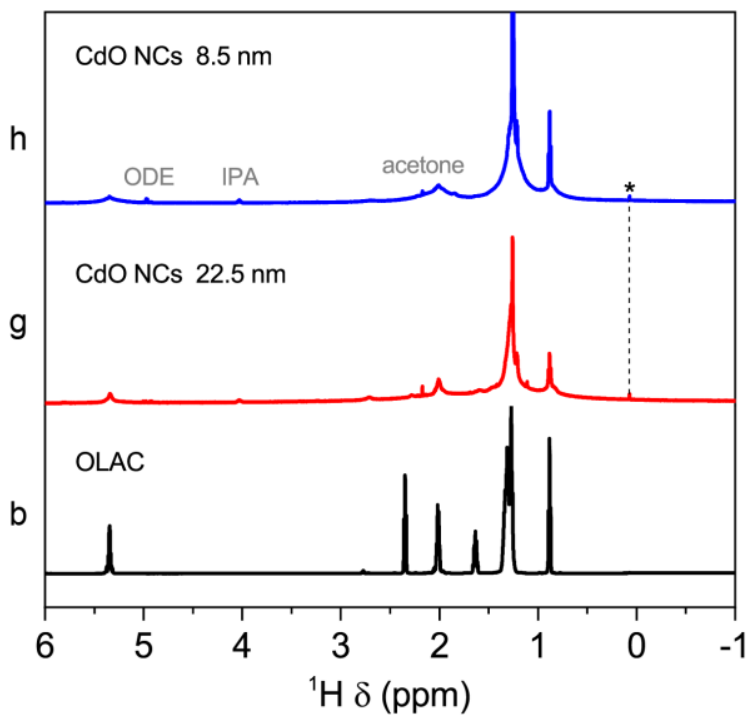

Peak B at $0.30 \mathrm{ppm}$

HMDS<smiles>[R]C(=O)O[Si](C)(C)C</smiles>

trimethylsilyl ester

Figure S6. NMR spectra of various reagents and solution mixtures. (a-c) NMR spectrum of OLAC (a), HMDS (b) and the reaction product between OLAC and HMDS (c). OLAC was degassed under vacuum for one hour before NMR measurement. (d) NMR spectrum of the mixture consisting of $\mathrm{Cd}(\mathrm{acac})_{2}$, OLAC and $\mathrm{ODE}$ after being degassed under vacuum for one hour at $100{ }^{\circ} \mathrm{C}$. (e) 
NMR spectrum of the mixture shown in (d) after adding HMDS and being kept at its reflux temperature $\left(\sim 320^{\circ} \mathrm{C}\right)$ for $10 \mathrm{~min}$. The color of solution studied in (e) turned dark brown after $\sim 30$ min of refluxing, indicating nucleation of $\mathrm{CdO}$ NCs. The reaction flask was air-cooled to room temperature after refluxing for $30 \mathrm{~min}$ and NMR spectrum of crude NC reaction mixture was shown in (f). (g, h) NMR spectrum of purified CdO NCs with an average size of (g) $22.5 \mathrm{~nm}$ and (h) $8.5 \mathrm{~nm}$. The peaks at 2.17, $4.04 \mathrm{ppm}$ and $4.97 \mathrm{ppm}$ are attributed to residual acetone, isopropanol (IPA) and ODE, respectively. The peak labelled as "*” is attributed to the vacuum grease introduced unintentionally to the reaction flask during $\mathrm{NC}$ synthesis. Both peaks at 0.30 and 0.18 ppm disappeared on the NMR spectrum of crude reaction mixtures (spectrum (f)), suggesting that trimethylsilyl ester likely decomposed upon completion of reaction. Furthermore, no spectral feature attributable to trimethylsilyl ester was found with purified $\mathrm{CdO}$ NCs $(\mathrm{g}, \mathrm{h})$.
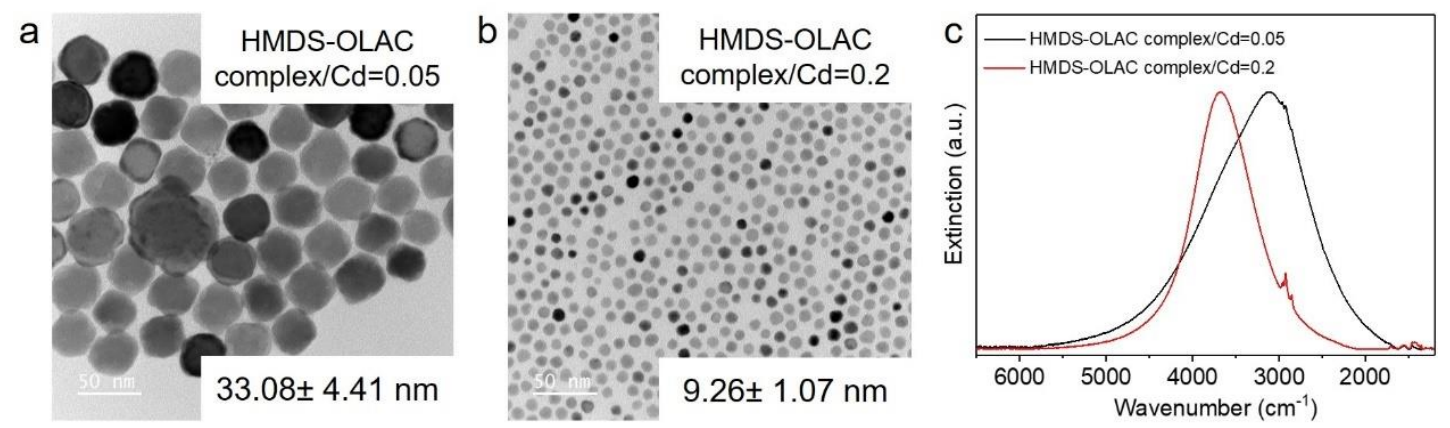

Figure S7. (a, b) TEM images and (c) extinction spectra of CdO NCs synthesized by replacing the additive HMDS with trimethylsilyl ester. The trimethylsilyl ester was synthesized as follows: 1:1 (molar ratio) mixture of OLA and HMDS was heated at $100{ }^{\circ} \mathrm{C}$ for one hour under $\mathrm{N}_{2}$. Then the mixture was vacuum degassed for another hour in order to remove unreacted HMDS (boiling point $=125^{\circ} \mathrm{C}$ ) and ammonia. This home-made trimethylsilyl was used as additive for CdO NC synthesis replacing HMDS. CdO NCs obtained showed nearly identical average size and LSPR energy as those synthesized from reactions utilizing HMDS. These results suggest that the trimethylsilyl ester is likely responsible for altered nucleation kinetics of CdO NCs. We postulate that the proposed mechanism remains valid for reactions that use LiHMDS, TTMSS, superhydride or TBAB since these additives are also known to react with OLAC to yield trimethylsilyl ester or borate ester. $^{24}$ 


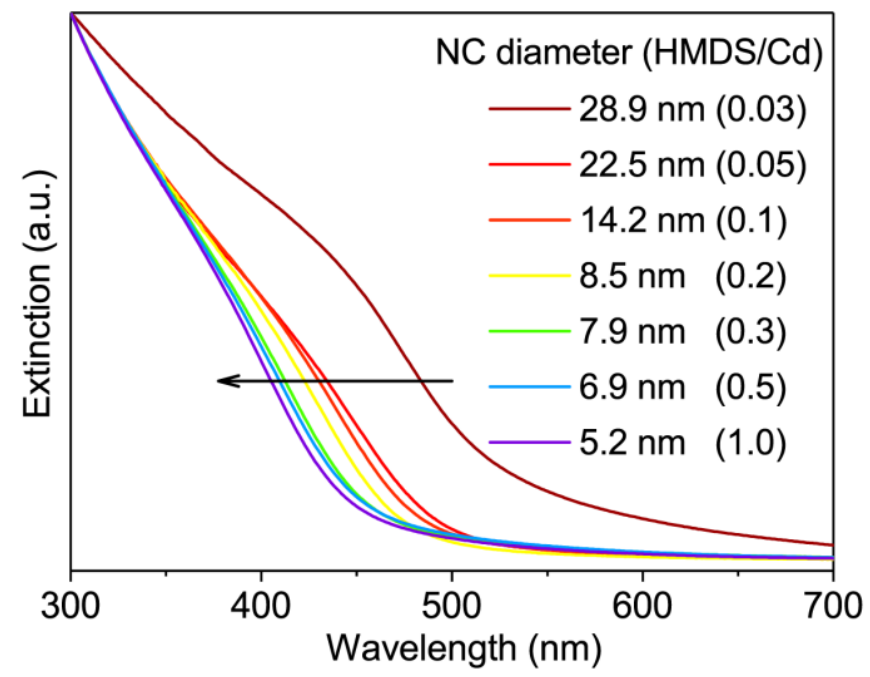

Figure S8. UV-Vis extinction spectra of CdO NCs synthesized using different HMDS/Cd molar ratios.

a
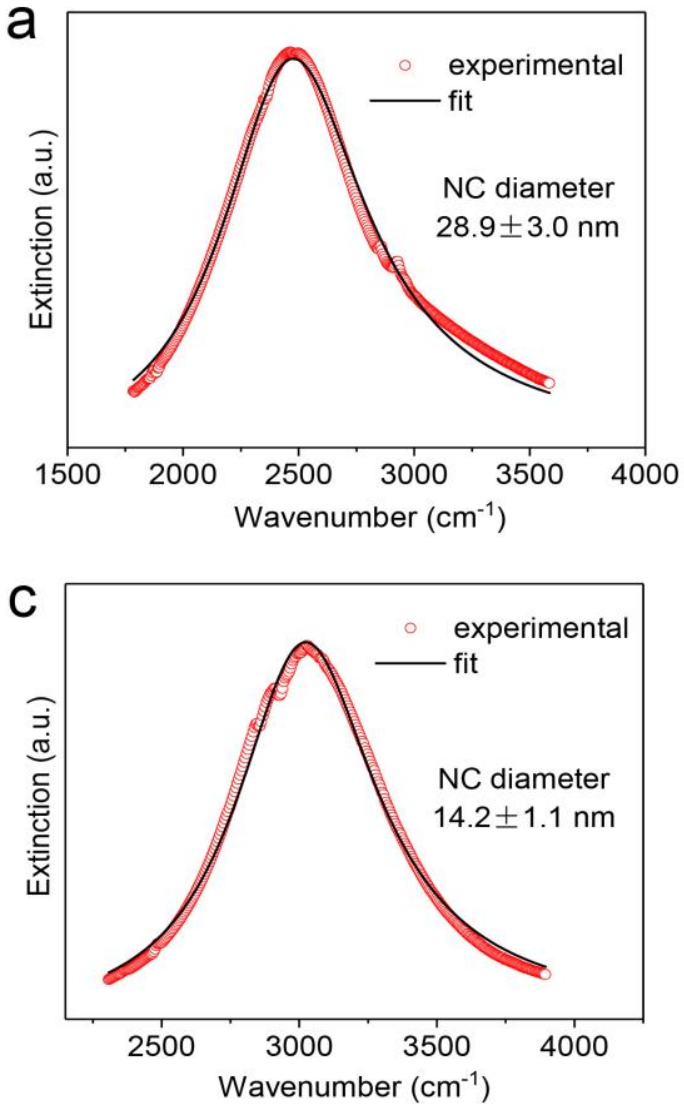
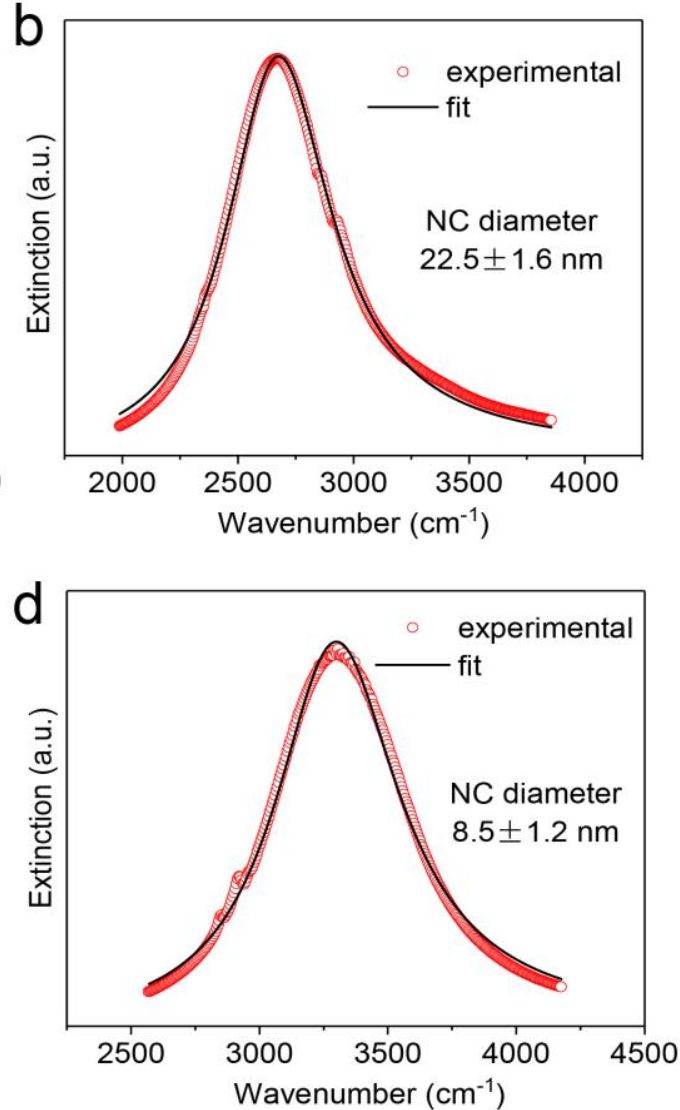

Figure S9. Examples showing Drude fitting of extinction spectra of CdO NCs synthesized using the HMDS/Cd molar ratio of (a) 0.03 , (b) 0.05 , (c) 0.1 , (d) 0.2 . 

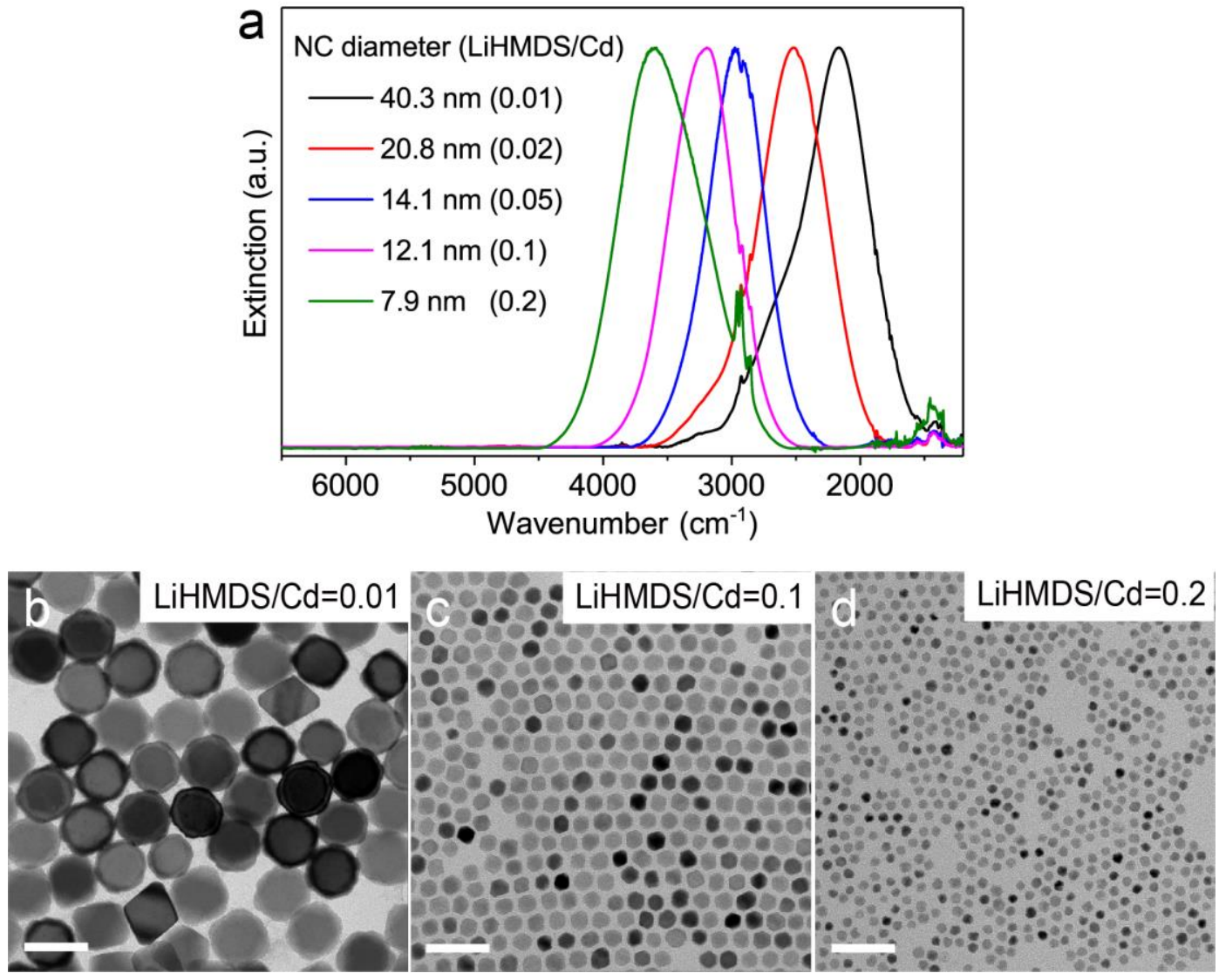

Figure S10. (a) Extinction spectra and (b-d) TEM images of CdO NCs synthesized using different LiHMDS/Cd molar ratios. Scale bars: $50 \mathrm{~nm}$. 

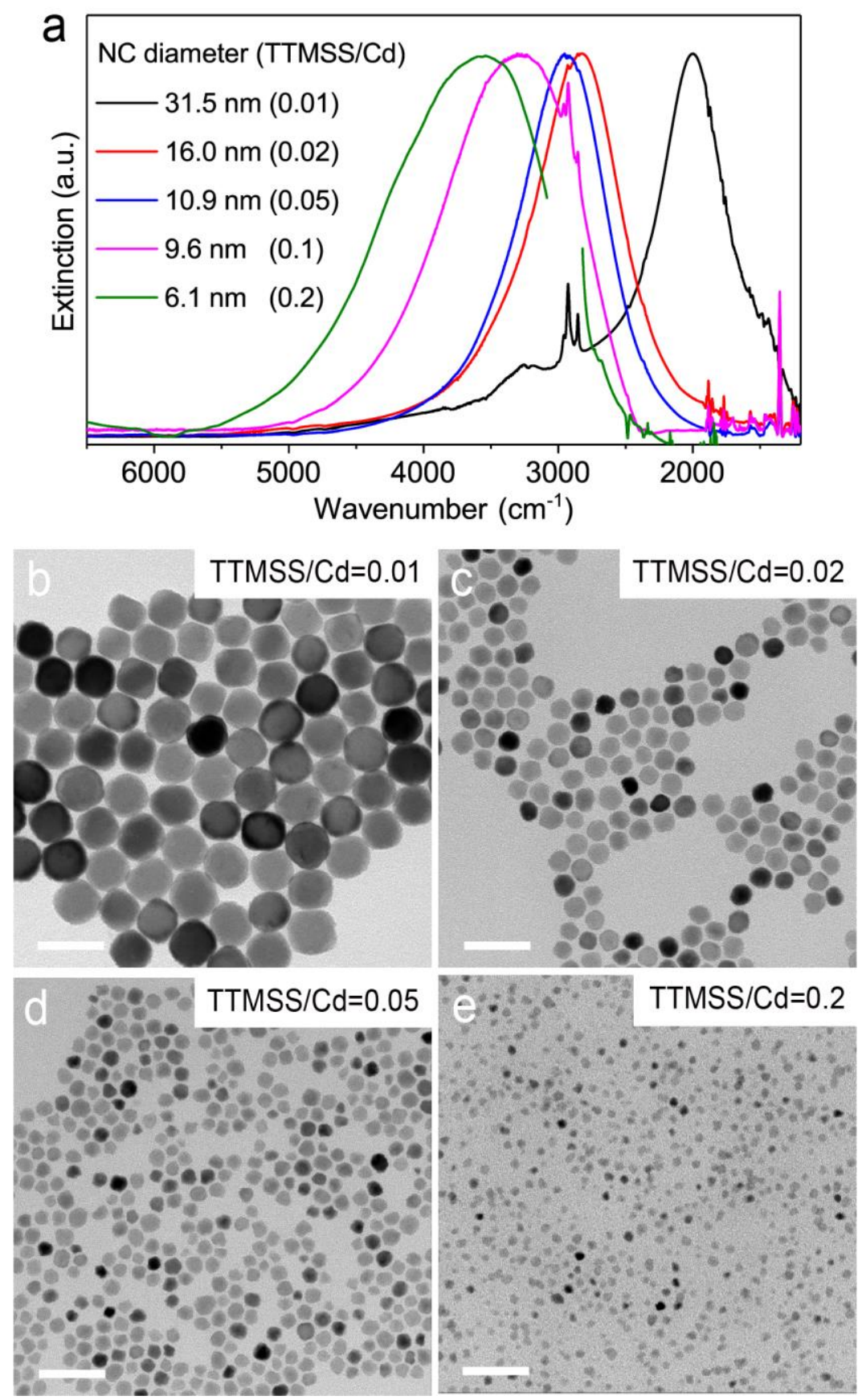

Figure S11. (a) Extinction spectra and (b-e) TEM images of CdO NCs synthesized using different TTMSS/Cd molar ratios. Scale bars: $50 \mathrm{~nm}$. 

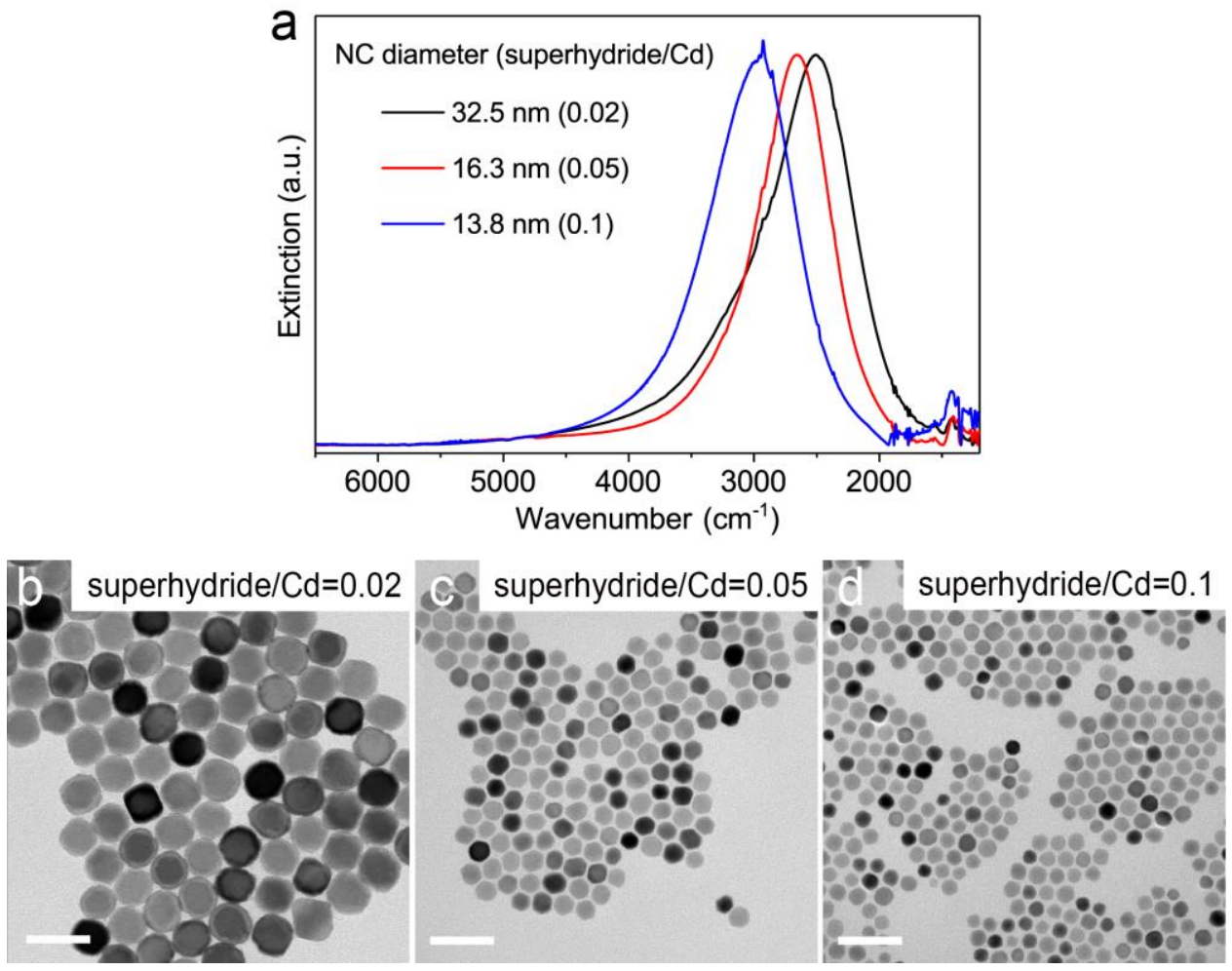

Figure S12. (a) Extinction spectra and (b-d) TEM images of CdO NCs synthesized using different superhydride/Cd molar ratios. Scale bars: $50 \mathrm{~nm}$.

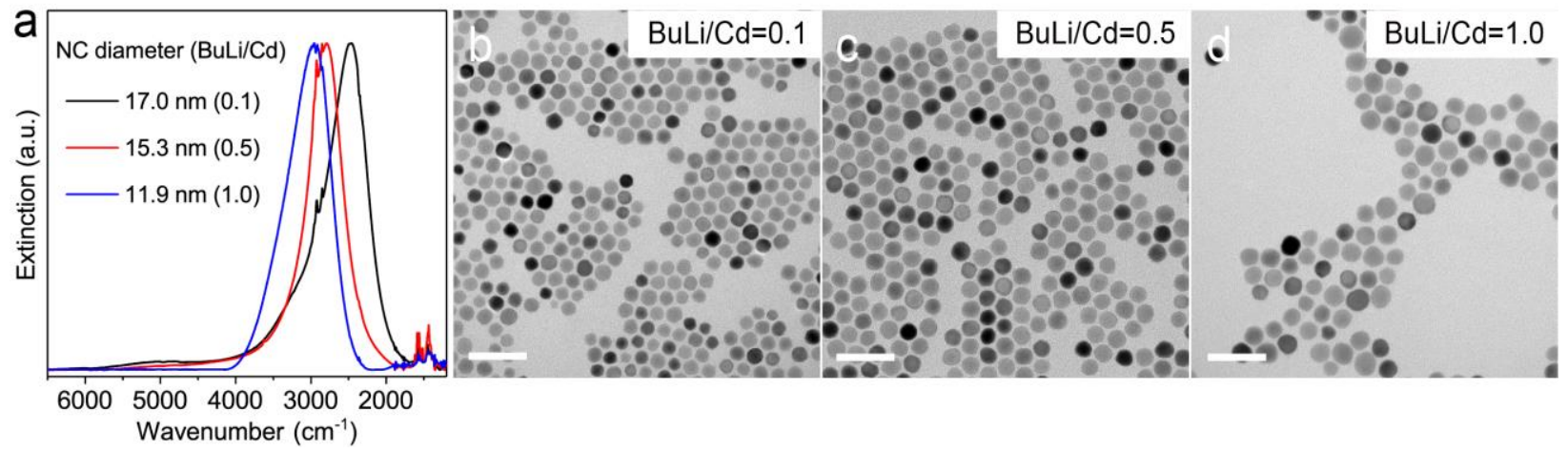

Figure S13. (a) Extinction spectra and (b-d) TEM images of CdO NCs synthesized using different $\mathrm{BuLi} / \mathrm{Cd}$ molar ratios. Scale bars: $50 \mathrm{~nm}$. 

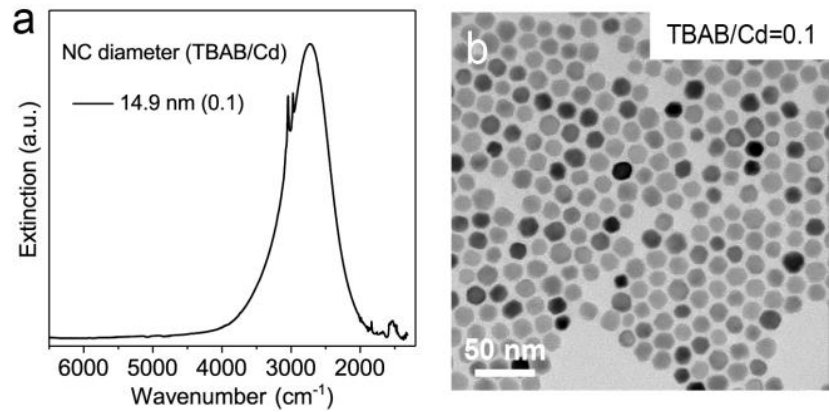

Figure S14. (a) Extinction spectra and (b) TEM image of CdO NCs synthesized using TBAB.

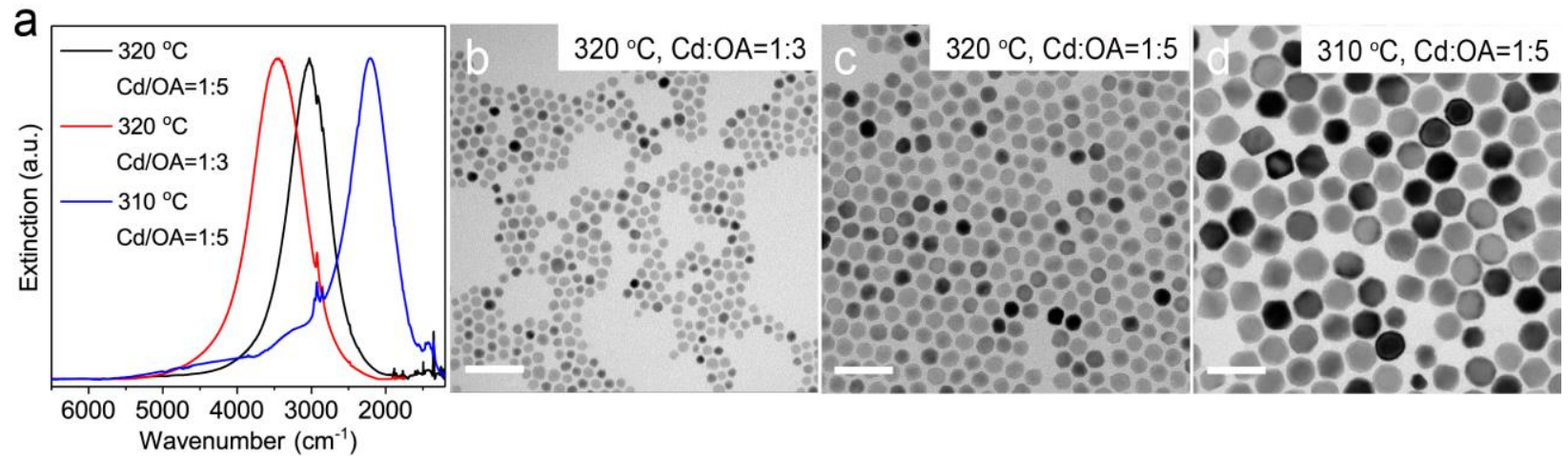

Figure S15. (a) Extinction spectra and (b-d) TEM images of CdO NCs synthesized using $\mathrm{HMDS} / \mathrm{Cd}=0.1$ at different temperatures and $\mathrm{Cd}$-to-OA molar ratios. The NC diameters are (b) $8.7 \pm 1.0 \mathrm{~nm}$, (c) $13.9 \pm 1.2 \mathrm{~nm}$ and (d) $24.6 \pm 2.4 \mathrm{~nm}$. Scale bars: $50 \mathrm{~nm}$.

Table S4. Summary of size and LSPR characteristics for CdO NCs synthesized using different additives.

\begin{tabular}{ccccccccccc}
\hline Additive & $\begin{array}{c}\text { Additive/Cd } \\
(\mathbf{m o l e} / \mathbf{m o l e})\end{array}$ & $\begin{array}{c}\boldsymbol{d} \\
(\mathbf{n m})\end{array}$ & $\begin{array}{c}\boldsymbol{\omega} \\
\left(\mathbf{c m}^{-1}\right)\end{array}$ & $\begin{array}{c}\lambda \\
(\mathbf{n m})\end{array}$ & $\begin{array}{c}\boldsymbol{E} \\
(\mathbf{e V})\end{array}$ & $\begin{array}{c}\Delta \boldsymbol{E} \\
(\mathbf{m e V})\end{array}$ & $\boldsymbol{Q}$ & $\begin{array}{c}\boldsymbol{\omega}_{\mathbf{p}} \\
\left(\mathbf{c m}^{-1}\right)\end{array}$ & $\begin{array}{c}\Gamma \\
\left(\mathbf{c m}^{-1}\right)\end{array}$ & $\begin{array}{c}\boldsymbol{N}_{\mathbf{e}} \\
\left(\mathbf{1 0}^{\mathbf{2 0}} \mathbf{c m}^{-3}\right)\end{array}$ \\
\hline \multirow{4}{*}{ HMDS } & 0.01 & $44.9 \pm 7.5$ & 1701 & 5880 & 0.211 & 122 & 1.73 & 5282 & 1371.6 & 0.84 \\
& 0.02 & $32.0 \pm 3.2$ & 1996 & 5010 & 0.248 & 63 & 3.94 & 6374 & 570.88 & 1.23 \\
& 0.03 & $28.9 \pm 3.0$ & 2467 & 4053 & 0.306 & 87 & 3.52 & 7848 & 757.93 & 1.86 \\
& $0.03-2$ & $27.4 \pm 4.1$ & 2250 & 4444 & 0.279 & 83 & 3.36 & 7135 & 630.71 & 1.54 \\
& 0.05 & $22.5 \pm 1.6$ & 2675 & 3738 & 0.332 & 69 & 4.81 & 8479 & 551.19 & 2.17 \\
& 0.2 & $14.2 \pm 1.1$ & 3035 & 3295 & 0.376 & 79 & 4.76 & 9579 & 621.75 & 2.77 \\
& $0.2-2$ & $11.2 \pm 2.4$ & 3339 & 2995 & 0.414 & 93 & 4.45 & 10551 & 678.88 & 3.36 \\
\hline
\end{tabular}




\begin{tabular}{|c|c|c|c|c|c|c|c|c|c|c|}
\hline & 0.3 & $7.9 \pm 1.1$ & 3596 & 2781 & 0.446 & 95 & 4.69 & 11290 & 737.46 & 3.85 \\
\hline & 0.5 & $6.9 \pm 1.5$ & 3880 & 2578 & 0.481 & 133 & 3.62 & 12186 & 929.38 & 4.49 \\
\hline & 1 & $5.2 \pm 1.4$ & 4159 & 2404 & 0.516 & 170 & 3.04 & 13390 & 1336.1 & 5.42 \\
\hline & $1-2$ & $5.9 \pm 1.5$ & 3839 & 2605 & 0.476 & 181 & 2.63 & 12153 & 1205.7 & 4.46 \\
\hline & $0.1^{*}$ & $8.7 \pm 1.0$ & 3452 & 2897 & 0.428 & 100 & 4.28 & 10875 & 716.17 & 3.57 \\
\hline & $0.1^{\&}$ & $24.6 \pm 2.4$ & 2202 & 4542 & 0.273 & 87 & 3.14 & 6971 & 641.12 & 1.47 \\
\hline \multirow{5}{*}{ LiHMDS } & 0.01 & $40.3 \pm 3.7$ & 2169 & 4610 & 0.269 & 80 & 3.36 & 6883 & 610.04 & 1.43 \\
\hline & 0.02 & $20.8 \pm 1.8$ & 2516 & 3974 & 0.312 & 78 & 4.00 & 7949 & 576.82 & 1.91 \\
\hline & 0.05 & $14.1 \pm 1.3$ & 2960 & 3379 & 0.367 & 65 & 5.65 & 9360 & 458.12 & 2.65 \\
\hline & 0.1 & $12.1 \pm 1.0$ & 3210 & 3116 & 0.398 & 73 & 5.45 & 10180 & 504.43 & 3.13 \\
\hline & 0.2 & $7.9 \pm 0.7$ & 3605 & 2774 & 0.447 & 98 & 4.56 & 11234 & 691.97 & 3.81 \\
\hline \multirow{5}{*}{ TTMSS } & 0.01 & $31.5 \pm 3.4$ & 2000 & 5000 & 0.248 & 87 & 2.85 & 6320 & 624.30 & 1.21 \\
\hline & 0.02 & $16.0 \pm 1.6$ & 2823 & 3543 & 0.350 & 102 & 3.43 & 8994 & 717.73 & 2.44 \\
\hline & 0.05 & $10.9 \pm 1.6$ & 2927 & 3416 & 0.363 & 96 & 3.78 & 9330 & 741.98 & 2.63 \\
\hline & 0.1 & $9.6 \pm 1.8$ & 3202 & 3123 & 0.397 & 150 & 2.65 & 10432 & 1110.7 & 3.29 \\
\hline & 0.2 & $6.1 \pm 1.7$ & 3556 & 2812 & 0.441 & 175 & 2.52 & 11400 & 1408.5 & 3.93 \\
\hline \multirow{3}{*}{$\begin{array}{l}\text { Superhy- } \\
\text { dride }\end{array}$} & 0.02 & $32.5 \pm 2.5$ & 2500 & 4000 & 0.310 & 106 & 2.92 & 8007 & 838.67 & 1.94 \\
\hline & 0.05 & $16.3 \pm 1.3$ & 2661 & 3758 & 0.330 & 87 & 3.79 & 8434 & 651.37 & 2.15 \\
\hline & 0.1 & $13.8 \pm 1.5$ & 2968 & 3370 & 0.368 & 102 & 3.61 & 9459 & 754.58 & 2.70 \\
\hline \multirow{3}{*}{ BuLi } & 0.1 & $17.0 \pm 2.4$ & 2476 & 4039 & 0.307 & 83 & 3.70 & 7961 & 652.16 & 1.92 \\
\hline & 0.5 & $15.3 \pm 1.5$ & 2815 & 3553 & 0.349 & 67 & 5.21 & 8877 & 493.75 & 2.38 \\
\hline & 1.0 & $11.9 \pm 1.2$ & 2944 & 3397 & 0.365 & 86 & 4.24 & 9498 & 608.76 & 2.73 \\
\hline TBAB & 0.1 & $14.9 \pm 1.3$ & 2710 & 3690 & 0.336 & 91 & 3.69 & 8681 & 683.27 & 2.28 \\
\hline
\end{tabular}

Note: $\omega$ : LSPR peak wavenumber, $\lambda$ : LSPR peak wavelength, $E$ : LSPR peak energy, $\Delta E$ : LSPR fwhm, $Q$ : LSPR quality factor $(E / \Delta E), \omega_{\mathrm{p}}$ : bulk plasma frequency, $\Gamma$ : free carrier damping constant, $N_{\mathrm{e}}$ : free carrier concentration. Unless otherwise specified, reactions were performed with $\mathrm{Cd} / \mathrm{OA}=1: 5$ at $320^{\circ} \mathrm{C}$.

${ }^{*}$ This sample was synthesized with $\mathrm{Cd} / \mathrm{OA}=1: 3$ at $320^{\circ} \mathrm{C}$.

${ }^{\&}$ This sample was synthesized with $\mathrm{Cd} / \mathrm{OA}=1: 5$ at $310^{\circ} \mathrm{C}$. 

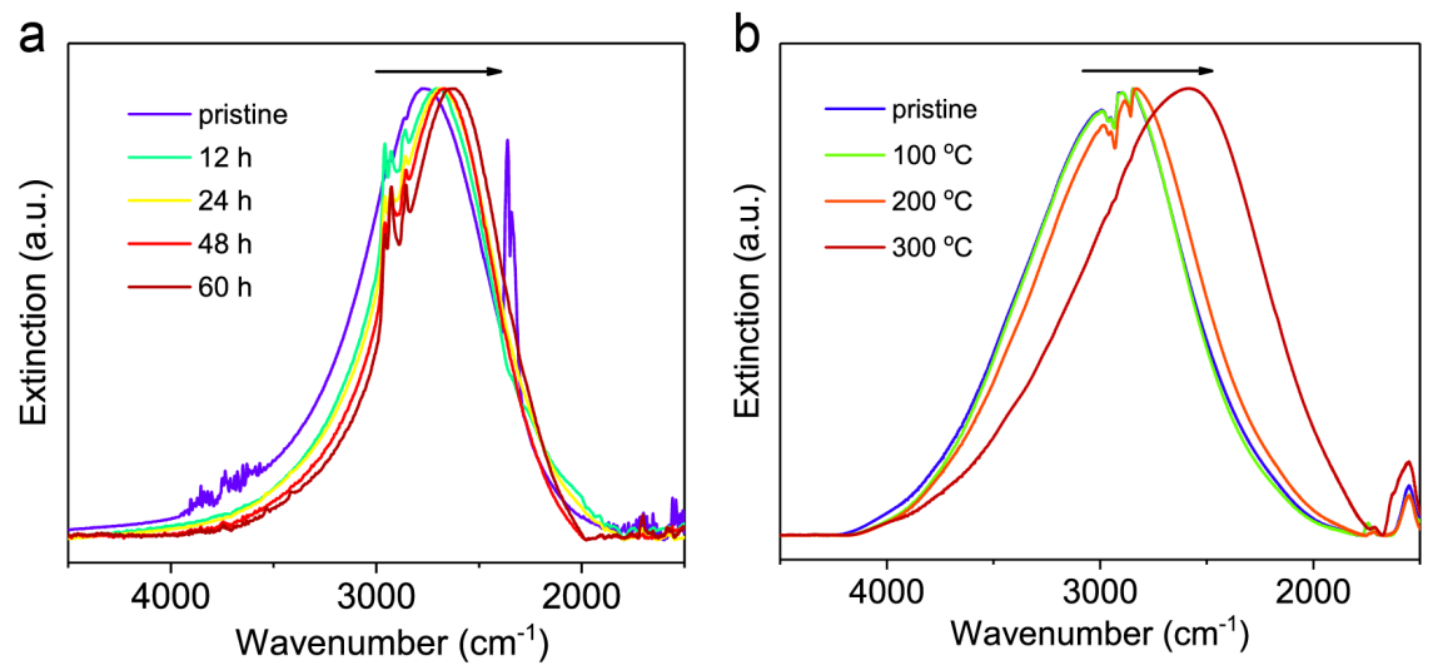

Figure S16. (a) Extinction spectra of CdO NCs (in hexane) that were left in air for different times. (b) Extinction spectra of $\mathrm{CdO} \mathrm{NC}$ thin film deposited on a $\mathrm{KBr}$ substrate after 10 minutes of thermal annealing at different temperatures as indicated on the graph.
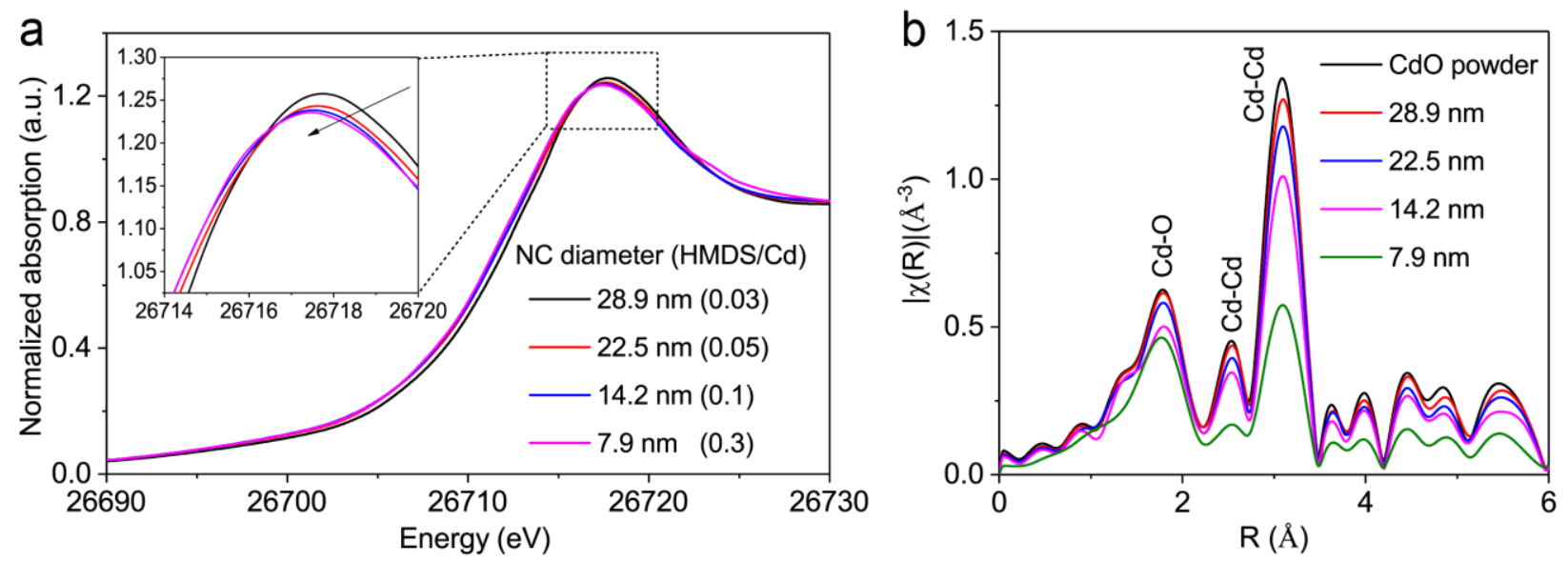

Figure S17. (a) XANES spectra and (b) Fourier transform of Cd $K$-edge EXAFS spectra of CdO NCs synthesized using different HMDS/Cd molar ratios. The EXAFS peak intensity is related to three parameters, namely coordination number $(N)$, the amplitude reduction factor $\left(S_{0}^{2}\right)$, and the Debye-Waller factor $\left(\sigma^{2}\right)$. The amplitude reduction factor is defined by the $\mathrm{Z}$ value, which was obtained by fitting the standard $\mathrm{Cd}$ data as 0.98 . The coordination number and Debye-Waller factor for each sample were obtained by fitting the experimental spectrum as shown in Table S5. In theory, the peak intensity would drop upon decreasing coordination number or increasing disorder of materials $\left(\sigma^{2}\right)$. The Debye-Waller factor is found to be essentially invariant for differently sized of CdO NCs. Consequently, the EXAFS peak intensity is largely dependent on the coordination number based on the fitting results of EXAFS spectra. 
Table S5. Local structural characteristics of CdO NCs determined by fitting the EXAFS data.

\begin{tabular}{|c|c|c|c|c|c|c|c|c|}
\hline $\begin{array}{c}\text { Diameter } \\
(\mathbf{n m})\end{array}$ & $\begin{array}{c}\text { HMDS/Cd } \\
\text { (mole/mole) }\end{array}$ & Shell & $N$ & $R(\AA)$ & $\begin{array}{c}\sigma^{2} \\
\left(\AA^{2}\right)\end{array}$ & $\begin{array}{c}\Delta E \\
(\mathrm{eV})\end{array}$ & $\begin{array}{c}R- \\
\text { Factor }\end{array}$ & $\begin{array}{c}\text { R-Range } \\
(\AA)\end{array}$ \\
\hline \multirow{2}{*}{$28.9 \pm 3.0$} & \multirow{2}{*}{0.03} & $\mathrm{Cd}-\mathrm{O}$ & 5.8 & 2.353 & 0.010 & 0.531 & \multirow{2}{*}{0.018} & \multirow{2}{*}{$1.20-3.47$} \\
\hline & & $\mathrm{Cd}-\mathrm{Cd}$ & 11.5 & 3.327 & 0.008 & 0.531 & & \\
\hline \multirow{2}{*}{$22.5 \pm 1.6$} & \multirow{2}{*}{0.05} & $\mathrm{Cd}-\mathrm{O}$ & 5.4 & 2.346 & 0.010 & 2.024 & \multirow{2}{*}{0.037} & \multirow{2}{*}{$1.45-3.48$} \\
\hline & & $\mathrm{Cd}-\mathrm{Cd}$ & 10.7 & 3.319 & 0.008 & 2.024 & & \\
\hline \multirow{2}{*}{$14.2 \pm 1.1$} & \multirow{2}{*}{0.1} & $\mathrm{Cd}-\mathrm{O}$ & 4.3 & 2.347 & 0.008 & 1.294 & \multirow{2}{*}{0.019} & \multirow{2}{*}{$1.20-3.46$} \\
\hline & & $\mathrm{Cd}-\mathrm{Cd}$ & 10.9 & 3.320 & 0.009 & 2.317 & & \\
\hline \multirow{2}{*}{$7.9 \pm 1.1$} & \multirow{2}{*}{0.3} & $\mathrm{Cd}-\mathrm{O}$ & 4.0 & 2.346 & 0.010 & 3.114 & \multirow{2}{*}{0.034} & \multirow{2}{*}{$1.20-3.48$} \\
\hline & & $\mathrm{Cd}-\mathrm{Cd}$ & 6.9 & 3.317 & 0.010 & 3.015 & & \\
\hline
\end{tabular}

Note: $N$ : Average coordination number, $R$ : path distance, $\sigma^{2}$ : Debye-Waller factor, $\Delta E$ : threshold energy correction, $R$-Range: $R$-space range for fitting. 

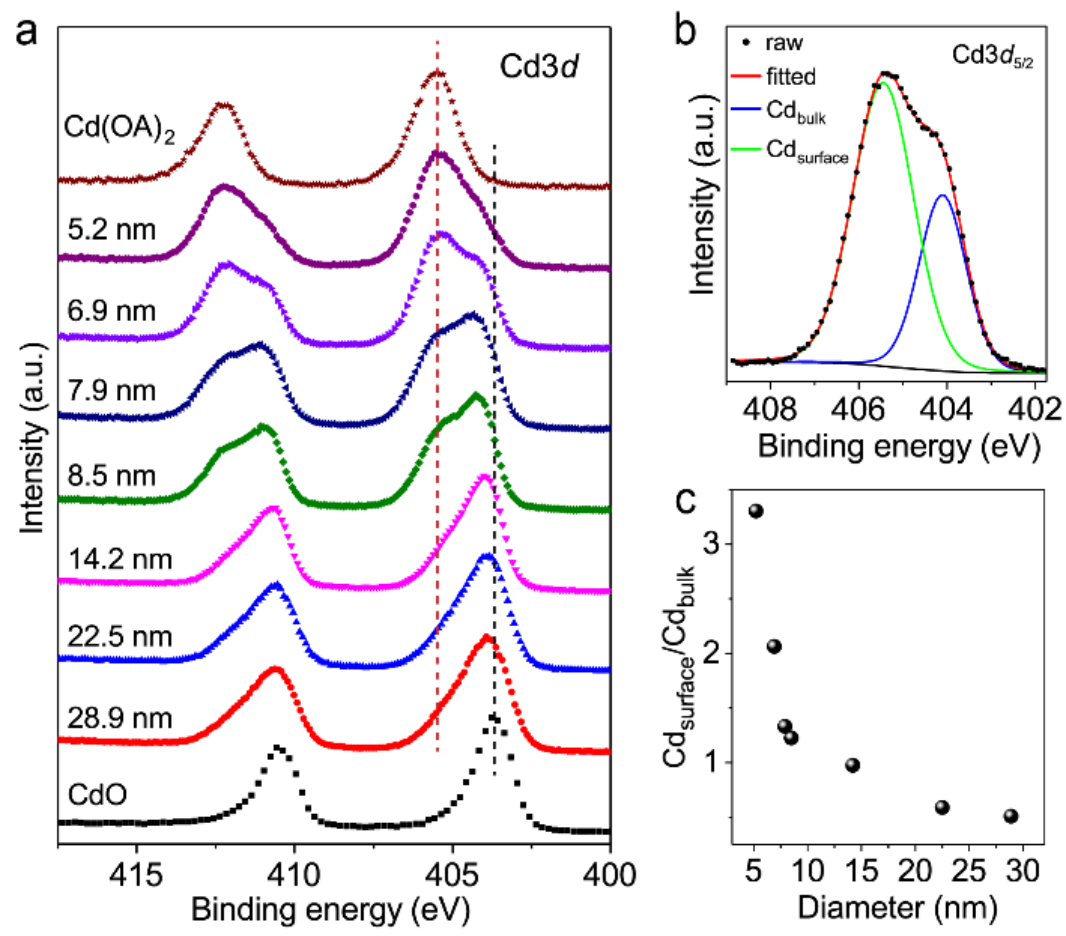

Figure S18. (a) High-resolution XPS spectra of $\mathrm{CdO}$ NCs in the $\mathrm{Cd} 3 d$ region. Cadmium oleate $\left(\mathrm{Cd}(\mathrm{OA})_{2}\right)$ and commercial $\mathrm{CdO}$ were used as reference compounds. (b) Deconvolution of the $\mathrm{Cd}$ $3 d_{5 / 2}$ XPS peak for $6.9 \mathrm{~nm}$ CdO NCs. (c) $\mathrm{Cd}_{\text {surface }} / \mathrm{Cd}_{\text {bulk }}$ ratios extracted from XPS peak deconvolution for $\mathrm{CdO}$ NCs of different sizes. The $\mathrm{Cd}_{\text {surface }}$ peak position remained essentially unchanged while the $\mathrm{Cd}_{\text {bulk }}$ shifted monotonically to higher binding energies for smaller CdO NCs. Similar size-dependent XPS peak shift was observed on PbS NCs, which was rationalized by considering increasing surface-to-volume ratio and thus relative amount of dangling bonds on $\mathrm{NC}$ surface as $\mathrm{NC}$ size decreases. ${ }^{25}$ 

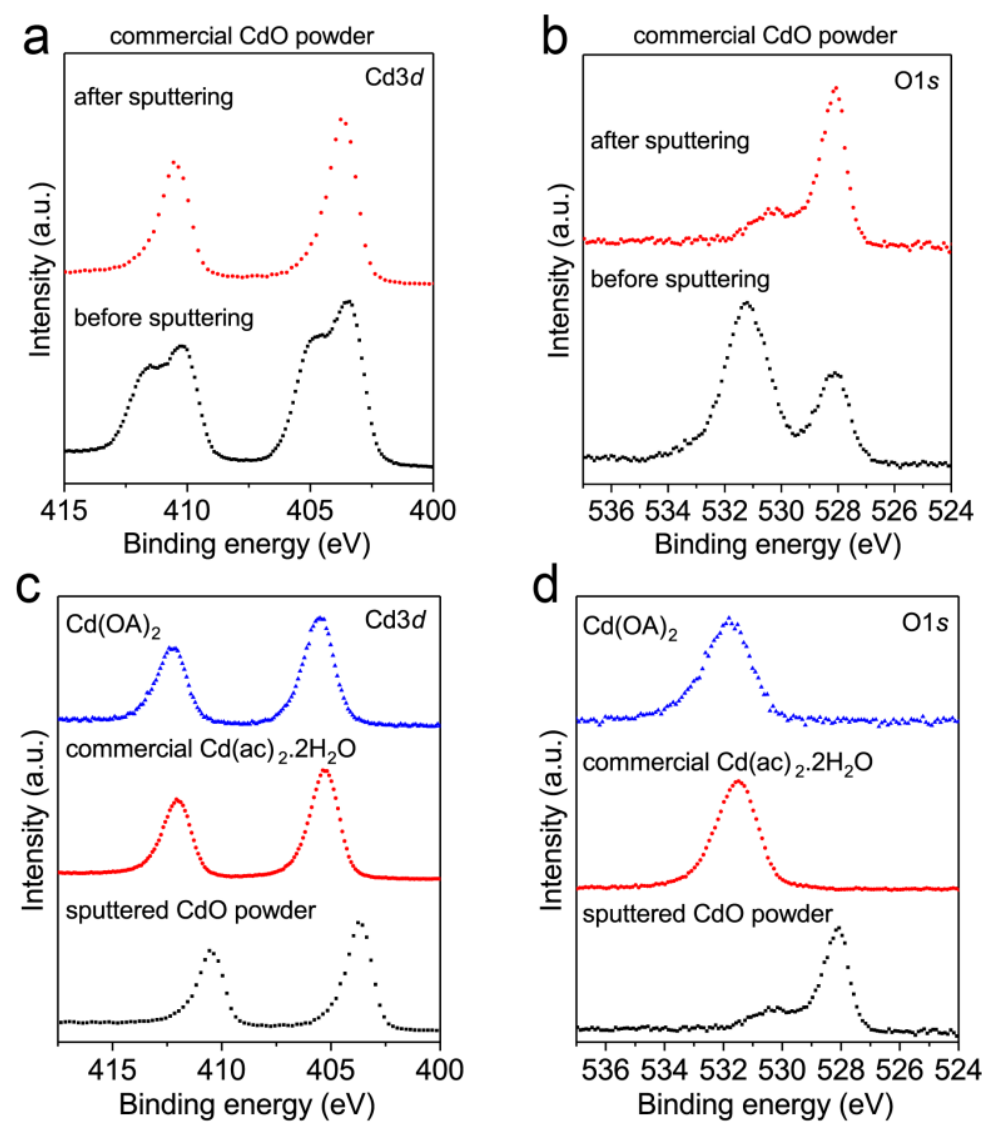

Figure S19. High-resolution XPS spectra of commercial CdO powder before and after sputtering in (a) $\mathrm{Cd} 3 d$ and (b) $\mathrm{O} 1 s$ regions. The sputtering process removes surface hydroxyl groups and allows the intrinsic structural characteristics of $\mathrm{CdO}$ powder to be probed. High-resolution XPS spectra of cadmium oleate $\left(\mathrm{Cd}(\mathrm{OA})_{2}\right)$, commercial $\mathrm{Cd}(\mathrm{ac})_{2} \cdot 2 \mathrm{H}_{2} \mathrm{O}$ and sputtered $\mathrm{CdO}$ in (c) $\mathrm{Cd} 3 d$ and (d) O1s regions . 
Table S6. Summary of peak deconvolution results for the XPS data shown in Figure S18a.

\begin{tabular}{|c|c|c|c|c|c|c|c|}
\hline $\begin{array}{c}\text { Diameter } \\
(\mathbf{n m})\end{array}$ & $\begin{array}{c}\text { HMDS/Cd } \\
\text { (mole/mole) }\end{array}$ & Type & $\begin{array}{c}\text { Peak energy } \\
(\mathrm{eV})\end{array}$ & $\begin{array}{c}\text { Peak fwhm } \\
(\mathrm{eV})\end{array}$ & $\begin{array}{l}\text { Peak } \\
\text { Area }\end{array}$ & $\begin{array}{c}\text { Peak Area } \\
(\%)\end{array}$ & $\begin{array}{c}\text { Cdsurface } \\
/ \text { Cd }_{\text {bulk }}\end{array}$ \\
\hline \multirow{2}{*}{$28.9 \pm 3.0$} & \multirow{2}{*}{0.03} & $\mathrm{Cd}_{\text {bulk }}$ & 403.83 & 1.43 & 8148 & 66.21 & \multirow{2}{*}{0.510} \\
\hline & & $\mathrm{Cd}_{\text {surface }}$ & 405.04 & 1.69 & 4159 & 33.79 & \\
\hline \multirow{2}{*}{$22.5 \pm 1.6$} & \multirow{2}{*}{0.05} & $\mathrm{Cd}_{\text {bulk }}$ & 403.85 & 1.38 & 7030 & 62.93 & \multirow{2}{*}{0.589} \\
\hline & & $\mathrm{Cd}_{\text {surface }}$ & 405.20 & 1.72 & 4141 & 37.07 & \\
\hline \multirow{2}{*}{$14.2 \pm 1.1$} & \multirow{2}{*}{0.1} & $\mathrm{Cd}_{\text {bulk }}$ & 403.88 & 1.13 & 6231 & 50.58 & \multirow{2}{*}{0.977} \\
\hline & & $\mathrm{Cd}_{\text {surface }}$ & 404.84 & 1.71 & 6088 & 49.42 & \\
\hline \multirow{2}{*}{$8.5 \pm 1.2$} & \multirow{2}{*}{0.2} & $\mathrm{Cd}_{\text {bulk }}$ & 404.06 & 1.15 & 4950 & 44.92 & \multirow{2}{*}{1.226} \\
\hline & & $\mathrm{Cd}_{\text {surface }}$ & 405.27 & 1.66 & 6070 & 55.08 & \\
\hline \multirow{2}{*}{$7.9 \pm 1.1$} & \multirow{2}{*}{0.3} & $\mathrm{Cd}_{\text {bulk }}$ & 404.11 & 1.32 & 4049 & 42.87 & \multirow{2}{*}{1.333} \\
\hline & & $\mathrm{Cd}_{\text {surface }}$ & 405.40 & 1.81 & 5396 & 57.13 & \\
\hline \multirow{2}{*}{$6.9 \pm 1.5$} & \multirow{2}{*}{0.5} & $\mathrm{Cd}_{\text {bulk }}$ & 404.10 & 1.26 & 2297 & 32.63 & \multirow{2}{*}{2.065} \\
\hline & & $\mathrm{Cd}_{\text {surface }}$ & 405.44 & 1.62 & 4741 & 67.37 & \\
\hline \multirow{2}{*}{$5.2 \pm 1.4$} & \multirow{2}{*}{1.0} & $\mathrm{Cd}_{\text {bulk }}$ & 404.11 & 1.37 & 1287 & 23.23 & \multirow{2}{*}{3.305} \\
\hline & & $\mathrm{Cd}_{\text {surface }}$ & 405.44 & 1.72 & 4254 & 76.77 & \\
\hline
\end{tabular}

Table S7. Summary of dopant efficiency of plasmonic metal oxide and Si NCs.

\begin{tabular}{ccccc}
\hline Host & Aliovalent dopant & $\begin{array}{c}\text { Doping effi- } \\
\text { ciency }\end{array}$ & $\begin{array}{c}\text { LSPR wave- } \\
\text { length range }\end{array}$ & Ref. \\
\hline $\mathrm{CdO}$ & N.A. & $\geqq 7 \%$ & $\sim 2.4-5.9 \mu \mathrm{m}$ & this work \\
$\mathrm{CdO}$ & $\mathrm{In}$ & $\sim 18 \%$ & $\sim 2 \mu \mathrm{m}$ & 26 \\
$\mathrm{In}_{2} \mathrm{O}_{3}$ & $\mathrm{Sn}$ & $\sim 11 \%$ & $\sim 1.9 \mu \mathrm{m}$ & 19 \\
$\mathrm{In}_{2} \mathrm{O}_{3}$ & $\mathrm{Sn}$ & $36 \%-71 \%$ & $\sim 1.6-2.5 \mu \mathrm{m}$ & 9 \\
$\mathrm{ZnO}$ & $\mathrm{Al}$ & $\sim 2 \%-5 \%$ & $>2.5 \mu \mathrm{m}$ & 27 \\
$\mathrm{ZnO}$ & $\mathrm{Al}$ & $20 \%-66 \%$ & $\sim 1.6-3.2 \mu \mathrm{m}$ & 17 \\
$\mathrm{Si}$ & $\mathrm{B}$ & $2.8 \%-5.2 \%$ & $\sim 2.0-3.2 \mu \mathrm{m}$ & 28 \\
$\mathrm{Si}$ & $\mathrm{P}$ & $0.3 \%-0.9 \%$ & $\sim 0.7-1.0 \mu \mathrm{m}$ & 28 \\
\hline
\end{tabular}




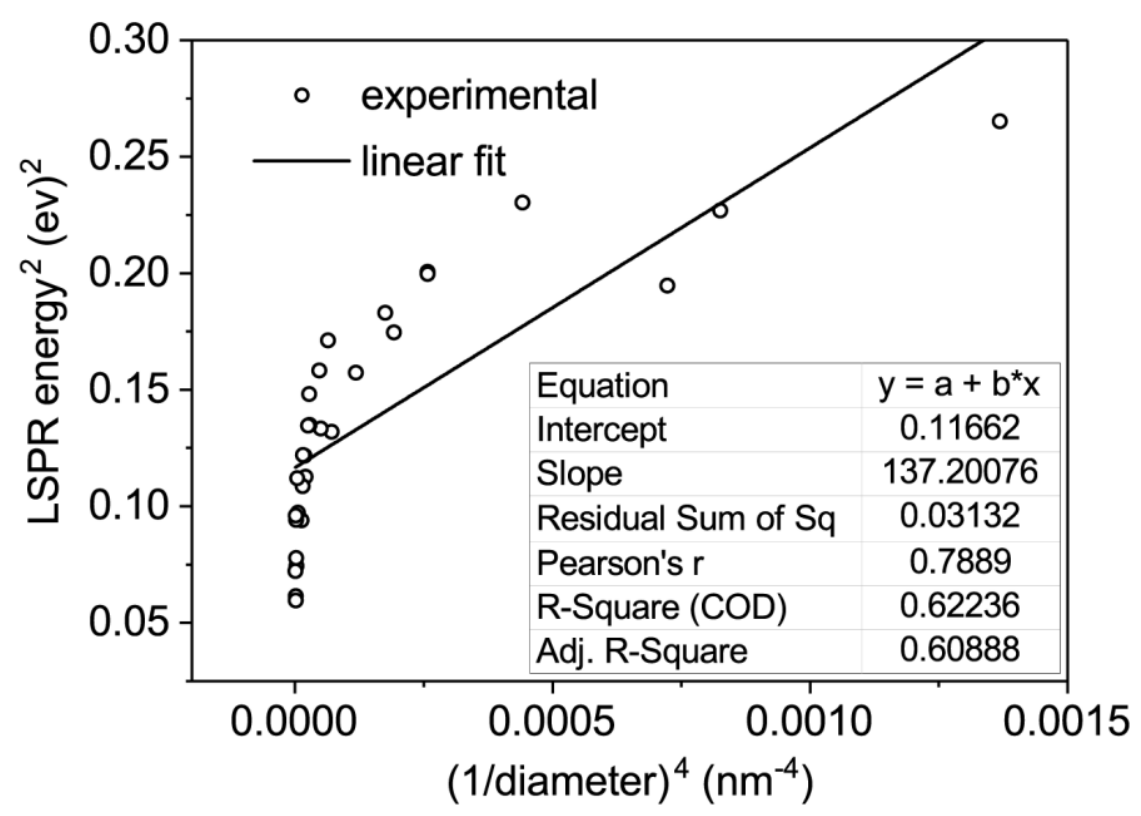

Figure S20. Fitting of experimental results according to equation (8) derived from the quantum plasmon model.

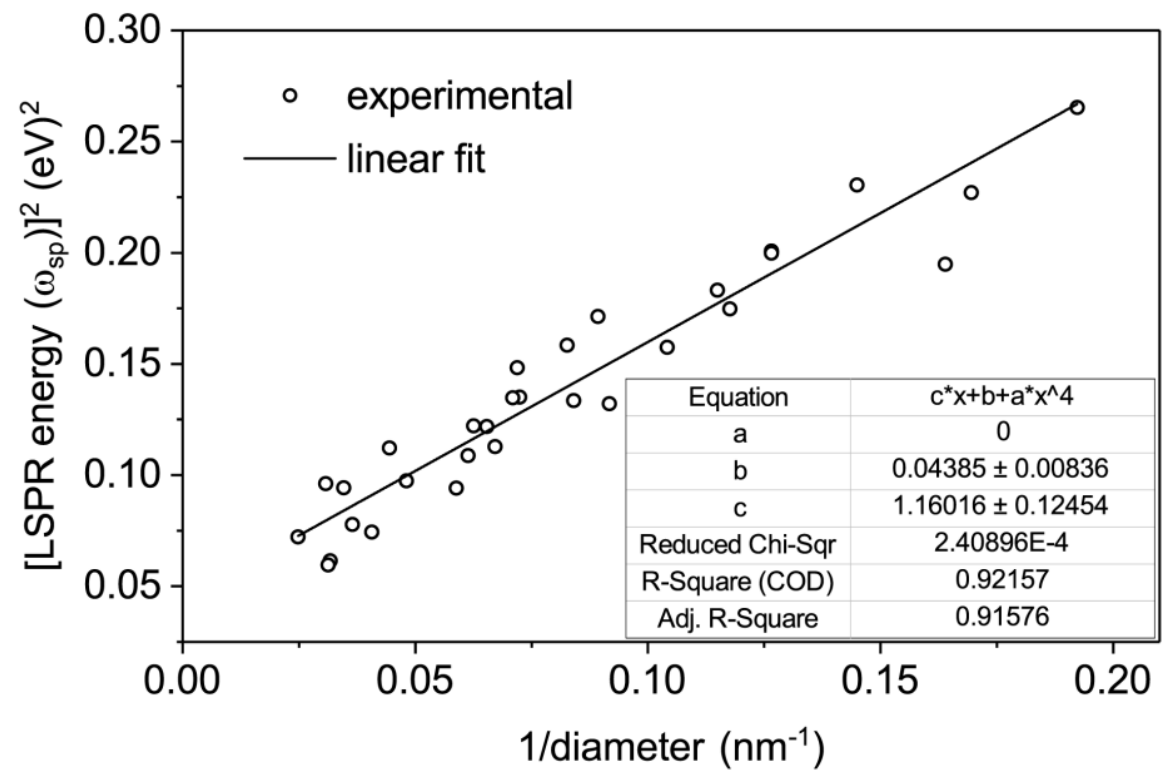

Figure S21. Linear fitting of the square of LSPR energy $\left(\omega_{\mathrm{sp}}\right)$ plotted against 1/diameter according to equation (13) derived from the hybrid size-dependent nonstoichiometry and quantum plasmon model. 


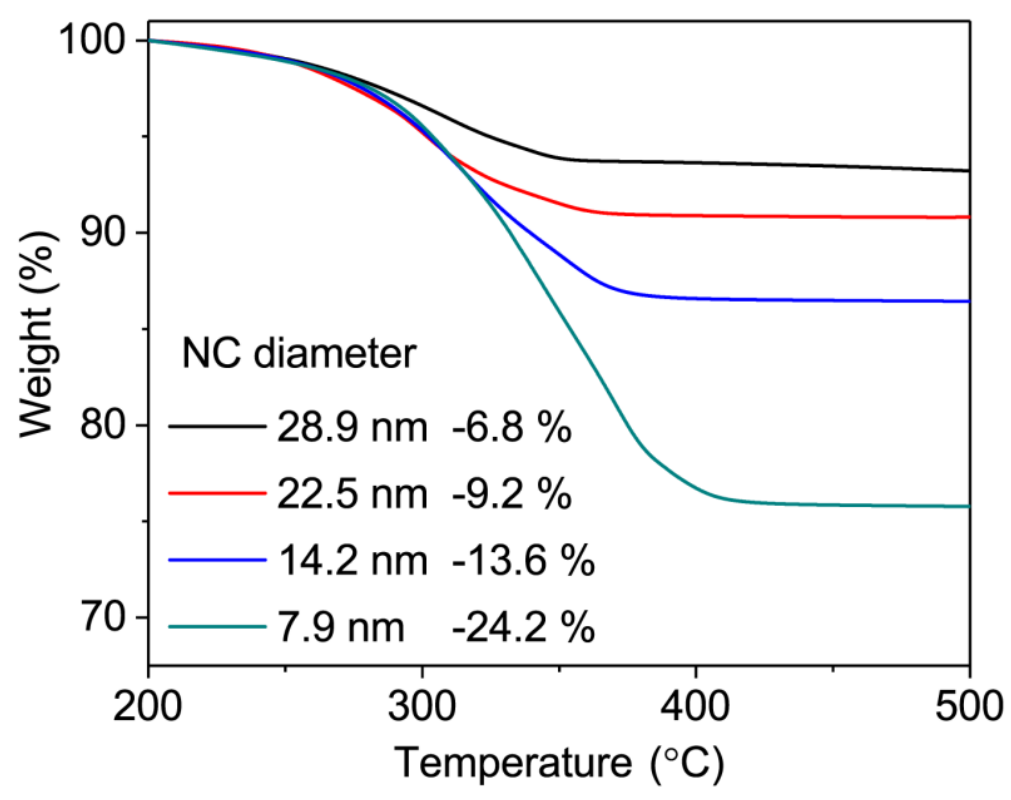

Figure S22. Thermogravimetric analysis (TGA) of CdO NCs synthesized using different $\mathrm{HMDS} / \mathrm{Cd}$ molar ratios.

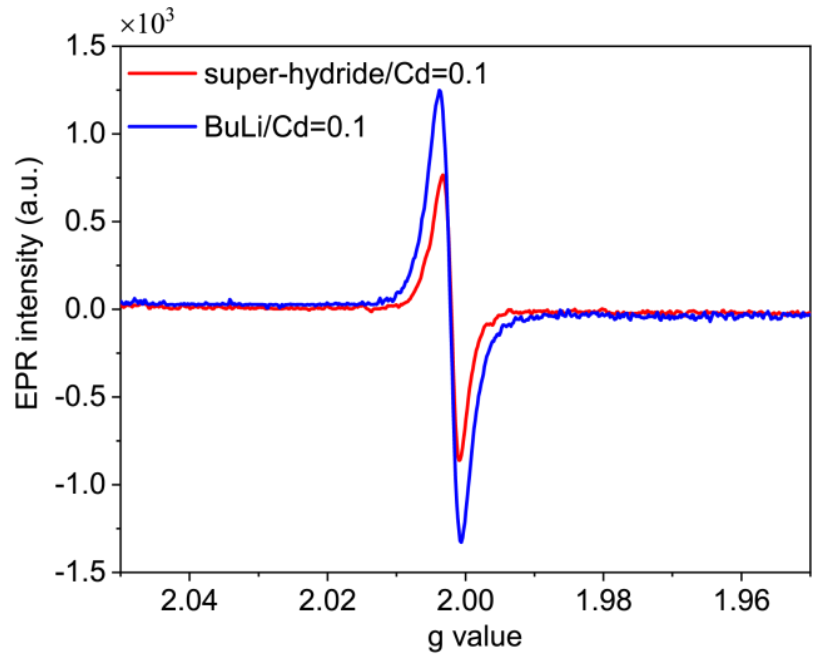

Figure S23. EPR spectra of CdO NCs synthesized using super-hydride and BuLi as the additive. 

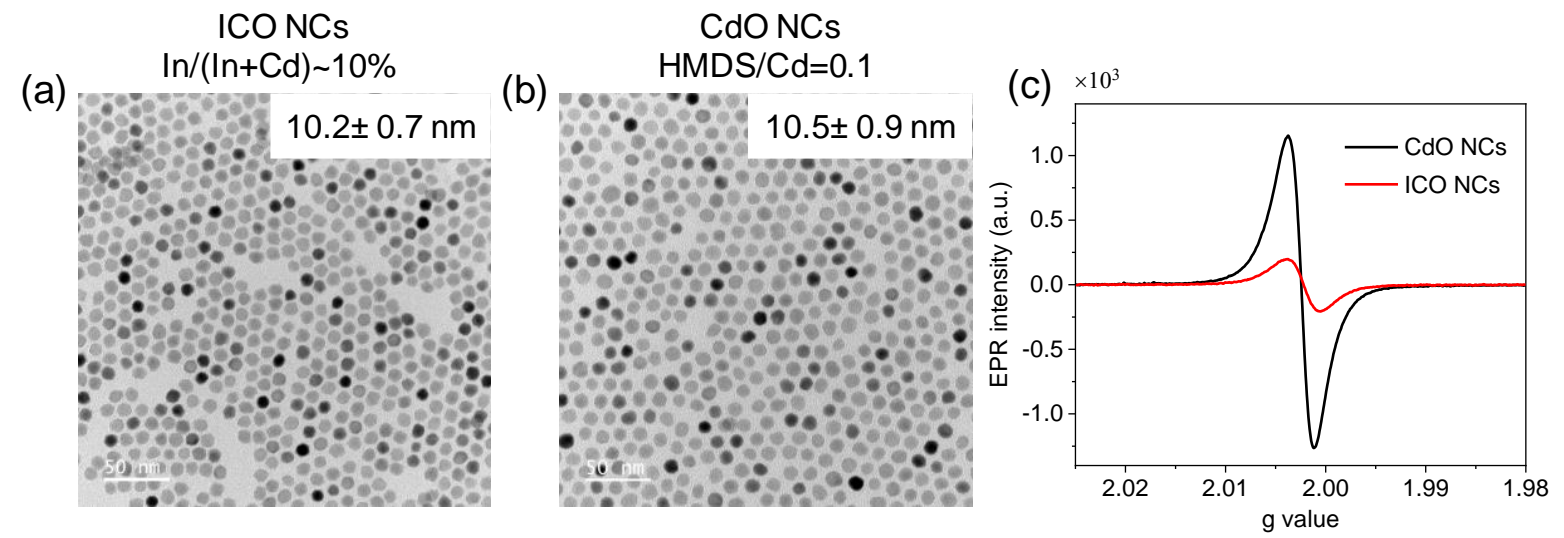

Figure S24. (a,b) TEM images and (c) EPR spectra of similarly sized CdO NCs and ICO NCs (with ca.10\% indium dopants). 


\section{Supplementary Reference}

(1) Nanda, J.; Sapra, S.; Sarma, D. D.; Chandrasekharan, N.; Hodes, G. Size-selected zinc sulfide nanocrystallites: Synthesis, structure, and optical studies. Chem. Mater. 2000, 12, 1018-1024.

(2) Borchert, H.; Shevchenko, E. V.; Robert, A.; Mekis, I.; Kornowski, A.; Grubel, G.; Weller, H. Determination of nanocrystal sizes: A comparison of TEM, SAXS, and XRD studies of highly monodisperse $\mathrm{CoPt}_{3}$ particles. Langmuir 2005, 21, 1931-1936.

(3) Natter, H.; Schmelzer, M.; Löffler, M. S.; Krill, C. E.; Fitch, A.; Hempelmann, R. Grain-growth kinetics of nanocrystalline iron studied in situ by synchrotron real-time X-ray diffraction. J. Phys. Chem. B 2000, 104, 2467-2476.

(4) Portales, H.; Goubet, N.; Saviot, L.; Adichtchev, S.; Murray, D.; Mermet, A.; Duval, E.; Pileni, M.-P. Probing atomic ordering and multiple twinning in metal nanocrystals through their vibrations. Proc. Natl. Acad. Sci. U.S.A. 2008, 105, 14784-14789.

(5) Liu, Z.; Zhong, Y.; Shafei, I.; Borman, R.; Jeong, S.; Chen, J.; Losovyj, Y.; Gao, X.; Li, N.; Du, Y.; Sarnello, E.; Li, T.; Su, D.; Ma, W.; Ye, X. Tuning infrared plasmon resonances in doped metal-oxide nanocrystals through cation-exchange reactions. Nat. Commun. 2019, 10, 1394.

(6) Dorfs, D.; Hartling, T.; Miszta, K.; Bigall, N. C.; Kim, M. R.; Genovese, A.; Falqui, A.; Povia, M.; Manna, L. Reversible tunability of the near-infrared valence band plasmon resonance in $\mathrm{Cu}_{2}$ xSe nanocrystals. J. Am. Chem. Soc. 2011, 133, 11175-11180.

(7) Sachet, E.; Shelton, C. T.; Harris, J. S.; Gaddy, B. E.; Irving, D. L.; Curtarolo, S.; Donovan, B. F.; Hopkins, P. E.; Sharma, P. A.; Sharma, A. L.; Ihlefeld, J.; Franzen, S.; Maria, J. P. Dysprosiumdoped cadmium oxide as a gateway material for mid-infrared plasmonics. Nat. Mater. 2015, 14, 414-420.

(8) Luther, J. M.; Jain, P. K.; Ewers, T.; Alivisatos, A. P. Localized surface plasmon resonances arising from free carriers in doped quantum dots. Nat. Mater. 2011, 10, 361-366.

(9) Lounis, S. D.; Runnerstrom, E. L.; Bergerud, A.; Nordlund, D.; Milliron, D. J. Influence of dopant distribution on the plasmonic properties of indium tin oxide nanocrystals. J. Am. Chem. Soc. 2014, 136, 7110-7116.

(10) Ueda, N.; Maeda, H.; Hosono, H.; Kawazoe, H. Band-gap widening of CdO thin films. $J$. Appl. Phys. 1998, 84, 6174-6177.

(11) Moreels, I.; Lambert, K.; De Muynck, D.; Vanhaecke, F.; Poelman, D.; Martins, J. C.; Allan, G.; Hens, Z. Composition and size-dependent extinction coefficient of colloidal PbSe quantum dots. Chem. Mater. 2007, 19, 6101-6106.

(12) Moreels, I.; Fritzinger, B.; Martins, J. C.; Hens, Z. Surface chemistry of colloidal PbSe nanocrystals. J. Am. Chem. Soc. 2008, 130, 15081-15086.

(13) Momma, K.; Izumi, F. VESTA 3 for three-dimensional visualization of crystal, volumetric and morphology data. J. Appl. Crystallogr. 2011, 44, 1272-1276.

(14) Zheng, B. J.; Lian, J. S.; Zhao, L.; Jiang, Q. Optical and electrical properties of In-doped CdO thin films fabricated by pulse laser deposition. Appl. Surf. Sci. 2010, 256, 2910-2914.

(15) Burbano, M.; Scanlon, D. O.; Watson, G. W. Sources of conductivity and doping limits in $\mathrm{CdO}$ from hybrid density functional theory. J. Am. Chem. Soc. 2011, 133, 15065-15072.

(16) Zhang, H.; Kulkarni, V.; Prodan, E.; Nordlander, P.; Govorov, A. O. Theory of quantum plasmon resonances in doped semiconductor nanocrystals. J. Phys. Chem. C 2014, 118, 1603516042 . 
(17) Greenberg, B. L.; Ganguly, S.; Held, J. T.; Kramer, N. J.; Mkhoyan, K. A.; Aydil, E. S.; Kortshagen, U. R. Nonequilibrium-plasma-synthesized $\mathrm{ZnO}$ nanocrystals with plasmon resonance tunable via $\mathrm{Al}$ doping and quantum confinement. Nano Lett. 2015, 15, 8162-8169.

(18) Schimpf, A. M.; Thakkar, N.; Gunthardt, C. E.; Masiello, D. J.; Gamelin, D. R. Chargetunable quantum plasmons in colloidal semiconductor nanocrystals. ACS Nano 2014, 8, 1065-1072. (19) Mendelsberg, R. J.; Garcia, G.; Li, H.; Manna, L.; Milliron, D. J. Understanding the plasmon resonance in ensembles of degenerately doped semiconductor nanocrystals. J. Phys. Chem. C 2012, $116,12226-12231$.

(20) Mendelsberg, R. J.; Zhu, Y.; Anders, A. Determining the nonparabolicity factor of the CdO conduction band using indium doping and the drude theory. J. Phys. D: Appl. Phys. 2012, 45, 425302.

(21) Lounis, S. D.; Runnerstrom, E. L.; Llordés, A.; Milliron, D. J. Defect chemistry and plasmon physics of colloidal metal oxide nanocrystals. J Phys. Chem. Lett. 2014, 5, 1564-1574.

(22) Kobylinski, M. M.; Ruhmlieb, C.; Kornowski, A.; Mews, A. Hexagonally shaped twodimensional Tin(II)sulfide nanosheets: Growth model and controlled structure formation. J. Phys. Chem. C 2018, 122, 5784-5795.

(23) Jereb, M.; Lakner, J. Synthesis of trimethylsilyl carboxylates by hmds under solvent-free conditions. Tetrahedron 2016, 72, 5713-5723.

(24) Brown, H. C.; Hébert, N. C. Organoboranes: XXXIII. Protonolysis of triethylborane with carboxylic acids. J. Organomet. Chem. 1983, 255, 135-142.

(25) Kovalev, A. I.; Wainstein, D. L.; Rashkovskiy, A. Y.; Osherov, A.; Golan, Y. Size shift of XPS lines observed from PbS nanocrystals. Surf. Interface Anal. 2010, 42, 850-854.

(26) Gordon, T. R.; Paik, T.; Klein, D. R.; Naik, G. V.; Caglayan, H.; Boltasseva, A.; Murray, C. B. Shape-dependent plasmonic response and directed self-assembly in a new semiconductor building block, indium-doped cadmium oxide (ICO). Nano Lett. 2013, 13, 2857-2863.

(27) Schimpf, A. M.; Ochsenbein, S. T.; Buonsanti, R.; Milliron, D. J.; Gamelin, D. R. Comparison of extra electrons in colloidal n-type $\mathrm{Al}^{3+}$-doped and photochemically reduced $\mathrm{ZnO}$ nanocrystals. Chem. Commun. 2012, 48, 9352-9354.

(28) Zhou, S.; Pi, X.; Ni, Z.; Ding, Y.; Jiang, Y.; Jin, C.; Delerue, C.; Yang, D.; Nozaki, T. Comparative study on the localized surface plasmon resonance of boron- and phosphorus-doped silicon nanocrystals. ACS Nano 2015, 9, 378-386. 